\title{
Nucleosomes around a mismatched base pair are excluded via an Msh2-dependent reaction with the aid of SNF2 family ATPase Smarcad1
}

\author{
Riki Terui, ${ }^{1,2}$ Koji Nagao, ${ }^{1,3}$ Yoshitaka Kawasoe, ${ }^{2}$ Kanae Taki, ${ }^{1}$ Torahiko L. Higashi, ${ }^{1,6}$ Seiji Tanaka, ${ }^{4,5}$ \\ Takuro Nakagawa, ${ }^{1}$ Chikashi Obuse, ${ }^{1,3}$ Hisao Masukata, ${ }^{1}$ and Tatsuro S. Takahashi ${ }^{2}$ \\ ${ }^{1}$ Graduate School of Science, Osaka University, Toyonaka, Osaka 560-0043, Japan; ${ }^{2}$ Faculty of Science, Kyushu University, \\ Nishi-ku, Fukuoka 819-0395, Japan; ${ }^{3}$ Graduate School of Life Science, Hokkaido University, Sapporo, Hokkaido 060-0810, Japan; \\ ${ }^{4}$ Division of Microbial Genetics, National Institute of Genetics, Mishima, Shizuoka 411-8540, Japan; ${ }^{5}$ School of Environmental \\ Science and Engineering, Kochi University of Technology, Kami-city, Kochi 782-8502, Japan
}

Post-replicative correction of replication errors by the mismatch repair (MMR) system is critical for suppression of mutations. Although the MMR system may need to handle nucleosomes at the site of chromatin replication, how MMR occurs in the chromatin environment remains unclear. Here, we show that nucleosomes are excluded from a $>1-k b$ region surrounding a mismatched base pair in Xenopus egg extracts. The exclusion was dependent on the Msh2-Msh6 mismatch recognition complex but not the Mlh1-containing MutL homologs and counteracts both the HIRA- and CAF-1 (chromatin assembly factor 1)-mediated chromatin assembly pathways. We further found that the Smarcad1 chromatin remodeling ATPase is recruited to mismatch-carrying DNA in an Msh2-dependent but Mlh1independent manner to assist nucleosome exclusion and that Smarcad1 facilitates the repair of mismatches when nucleosomes are preassembled on DNA. In budding yeast, deletion of FUN30, the homolog of Smarcad1, showed a synergistic increase of spontaneous mutations in combination with $\mathrm{MSH6}$ or MSH3 deletion but no significant increase with MSH2 deletion. Genetic analyses also suggested that the function of Fun30 in MMR is to counteract CAF-1. Our study uncovers that the eukaryotic MMR system has an ability to exclude local nucleosomes and identifies Smarcad1/Fun30 as an accessory factor for the MMR reaction.

[Keywords: mismatch repair; chromatin; nucleosome; Xenopus egg extract; yeast]

Supplemental material is available for this article.

Received December 19, 2017; revised version accepted April 27, 2018.

Precise DNA replication relies on both the fidelity of DNA synthesis by DNA polymerases and post-replicative correction of replication errors by the mismatch repair (MMR) system. MMR is critical for the suppression of tumorigenesis, as inactivation of MMR genes gives rise to a high risk of hereditary and sporadic cancers (Lynch et al. 2015). Post-replicative MMR occurs through the following steps: mismatch recognition, searching for strand discrimination signals, nicking on the newly synthesized DNA strand, degradation of the strand, and resynthesis of the removed segment (for reviews, see Iyer et al. 2006; Jiricny 2013; Kunkel and Erie 2015). Replication errors are recognized by two Msh2-containing complexes: MutSa (Msh2-

\footnotetext{
${ }^{6}$ Present address: Chromosome Segregation Laboratory, The Francis Crick Institute, London NW1 1AT, UK.

Corresponding author: tatsuro_takahashi@kyudai.jp

Article published online ahead of print. Article and publication date are online at http://www.genesdev.org/cgi/doi/10.1101/gad.310995.117. Freely available online through the Genes \& Development Open Access option.
}

Msh6) and MutS $\beta$ (Msh2-Msh3). The substrate specificities of the two complexes are partially overlapped, especially on small insertion/deletion loops (IDLs), although MutS $\alpha$ exhibits a preference for base-base mismatches and small IDLs, and MutS $\beta$ exhibits a preference for large IDLs (Marsischky et al. 1996; Genschel et al. 1998). In mammalian cells, MutSa is much more abundant than MutS $\beta$ and is primarily responsible for repair of basebase mismatches and small IDLs (Drummond et al. 1997; Genschel et al. 1998; Marra et al. 1998). Mismatchbound MutS $\alpha$ and MutS $\beta$ change their conformation to sliding clamps, recruit the MutLa endonuclease (Mlh1Pms2 in vertebrates), and translocate along DNA (for reviews, see Iyer et al. 2006; Jiricny 2013; Lee et al. 2014; Kunkel and Erie 2015). This reaction is likely required for the search for strand discrimination signals such as

(C) 2018 Terui et al. This article, published in Genes \& Development, is available under a Creative Commons License (Attribution 4.0 International), as described at http://creativecommons.org/licenses/by/4.0/. 
ssDNA breaks, repair intermediates of ribonucleotides embedded in the leading strand, and DNA-bound proliferating cell nuclear antigen (PCNA) (Holmes et al. 1990; Thomas et al. 1991; Pluciennik et al. 2010; Ghodgaonkar et al. 2013; Lujan et al. 2013; Kawasoe et al. 2016). Communication between MutLa, MutSa/MutS $\beta$, and DNA-bound PCNA induces strand-specific nicking by the MutLa endonuclease (Kadyrov et al. 2006; Pluciennik et al. 2010, 2013). A mismatch-containing segment is then degraded mainly by Exonuclease 1 (Exol) (Tishkoff et al. 1997; Amin et al. 2001). Finally, DNA polymerases resynthesize the degraded segment to complete the repair reaction.

Most DNA transactions occurring on chromatin require the movement, exchange, or displacement of nucleosomes (for reviews, see Ransom et al. 2010; Narlikar et al. 2013; Polo and Almouzni 2015). Histone chaperone CAF-1 (chromatin assembly factor 1) and HIRA support DNA synthesis-coupled and synthesis-independent chromatin assembly, respectively (Smith and Stillman 1989; Gaillard et al. 1996; Ray-Gallet et al. 2002). FACT (facilitates chromatin transcription) promotes the exchange of histones, particularly $\mathrm{H} 2 \mathrm{~A}-\mathrm{H} 2 \mathrm{~B}$ dimers, at the site of transcription, replication, and repair (for review, see Formosa 2012). Some DNA repair reactions are assisted by a specific class of adenosine triphosphate (ATP)-dependent chromatin remodeling enzyme (chromatin remodeler) (for review, see Narlikar et al. 2013). An example is SNF2-type chromatin remodeling enzyme Smarcad1, which facilitates longrange resection of double-strand break ends in the context of chromatin in both humans and yeast (Chen et al. 2012; Costelloe et al. 2012; Eapen et al. 2012; Densham et al. 2016). In addition to double-strand break end processing, Smarcad1 is involved in heterochromatin silencing (Neves-Costa et al. 2009; Rowbotham et al. 2011; Stralfors et al. 2011; Taneja et al. 2017). This factor is also enriched on the nascent DNA at the replication fork (Rowbotham et al. 2011; Sirbu et al. 2013) and is physically associated with Msh2-containing complexes (Okazaki et al. 2008; Rowbotham et al. 2011; Chen et al. 2016), yet the significance of these observations remains to be elucidated.

Accumulating evidence suggests that nucleosomes are assembled immediately behind the replication fork (McKnight and Miller 1977; Sogo et al. 1986; Lucchini and Sogo 1995; Shibahara and Stillman 1999; Smith and Whitehouse 2012), and thus post-replicative MMR may need to contend with nucleosomes to carry out its function in cells. On the one hand, both eukaryotic MutLa and bacterial MutL form a large proteinaceous ring that can rapidly diffuse along DNA, and eukaryotic MutLa can hop over nucleosomes (Gorman et al. 2010; Liu et al. 2016). Therefore, chromatin structure may not prevent the communication between MutL $\alpha$ and PCNA. MutS $\beta$ can also jump over nucleosomes (Brown et al. 2016), and the MutS $\beta$-dependent step in MMR could also function on chromatin. On the other hand, both single-molecule and biochemical studies demonstrated that nucleosomes are inhibitory for diffusion of MutSa along DNA /Gorman et al. 2010; Brown et al. 2016) and the MutSa-dependent MMR reaction (Li et al. 2009, 2013; Schopf et al. 2012). A possible means to assist a MutSa-dependent reaction on chromatin may simply be to localize it close to the replication fork, where nucleosomes must be transiently disassembled. MutSa is tethered to the replication machinery through its conserved PCNA-interacting motif (Kleczkowska et al. 2001; Hombauer et al. 2011; Haye and Gammie 2015) and to nucleosomes containing K36-methylated histone $\mathrm{H} 3$ through its PWWP motif, found in vertebrates (Li et al. 2013). Another possible means is to exclude or disassemble nucleosomes near replication errors. In vitro reconstitution studies have shown that human MutSa exhibits chromatin remodeling activity (Javaid et al. 2009) and that it competes with CAF-1-dependent chromatin assembly (Kadyrova et al. 2011; Schopf et al. 2012; Rodriges Blanko et al. 2016). Two chromatin-related factors, HMGB1 and regulatory factor X, are also reported to stimulate MMR in vitro (Yuan et al. 2004; Zhang et al. 2008), although their involvement in MMR in vivo has not been established. Despite the progress, however, how nucleosomes are handled by the MMR machinery during the repair reaction remains largely elusive. Furthermore, while many DNA repair reactions are assisted by chromatin remodelers and histone chaperones (Ransom et al. 2010; Narlikar et al. 2013; Polo and Almouzni 2015), it remains unclear whether the MMR system receives assistance from such factors in vivo.

Using Xenopus egg extracts as a model system, we studied how the MMR system handles nucleosomes after the recognition of a mismatch. We show here that nucleosomes around a mispaired base are efficiently excluded via an Msh2-dependent reaction. We further show that Smarcad1 is recruited to mismatch-carrying DNA depending on Msh2, assists nucleosome exclusion, and facilitates the repair of mismatches when nucleosomes are preassembled on DNA. Genetic experiments in yeast provide evidence that the homolog of Smarcad1 has a mutation suppressor function that is epistatic to Msh2 and antagonizes CAF-1. Our results reveal a dynamic interplay between MMR and chromatin and identify Smarcad1/Fun30 as a factor that assists the MMR reaction.

\section{Results}

Nucleosomes are excluded from $a>1-k b$ region surrounding a mismatch in Xenopus egg extracts

To study MMR in the context of chromatin, we used extracts of Xenopus eggs, which efficiently recapitulate DNA synthesis, MMR (Olivera Harris et al. 2015; Kawasoe et al. 2016), and both DNA synthesis-independent (HIRA-mediated) (Ray-Gallet et al. 2002) and synthesiscoupled (CAF-1-mediated) chromatin assembly (Gaillard et al. 1996) in vitro. Deposition of a nucleosome induces approximately one compensatory positive supercoil in closed circular duplexes, and by relaxing this torsional strain, topoisomerase I reduces the linking number of a plasmid by one for each nucleosome assembled. Upon incubation in the nucleoplasmic extract (NPE) of Xenopus eggs (Walter et al. 1998), a 3.0-kb closed circular plasmid $\left(\mathrm{pMM}{ }^{\text {homo }}\right.$ ) (Fig. 1A) became supercoiled within 2- 
Terui et al.

A

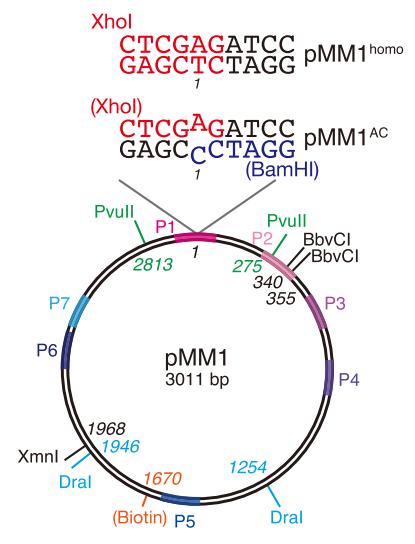

C



B

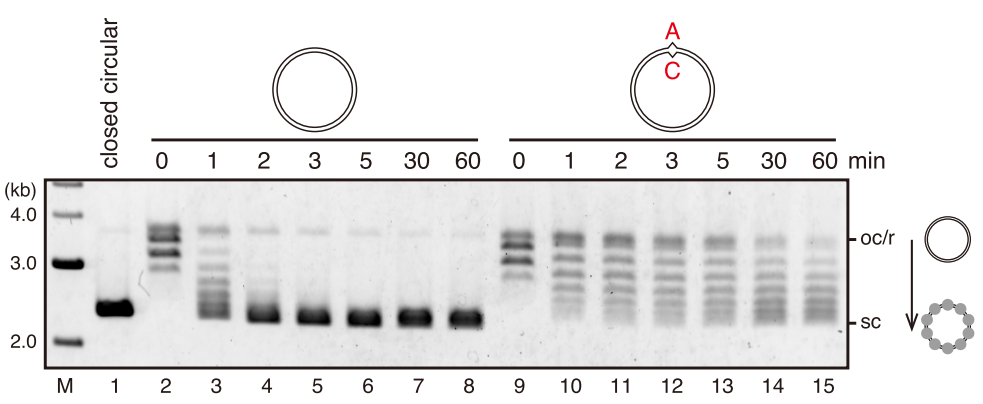

D
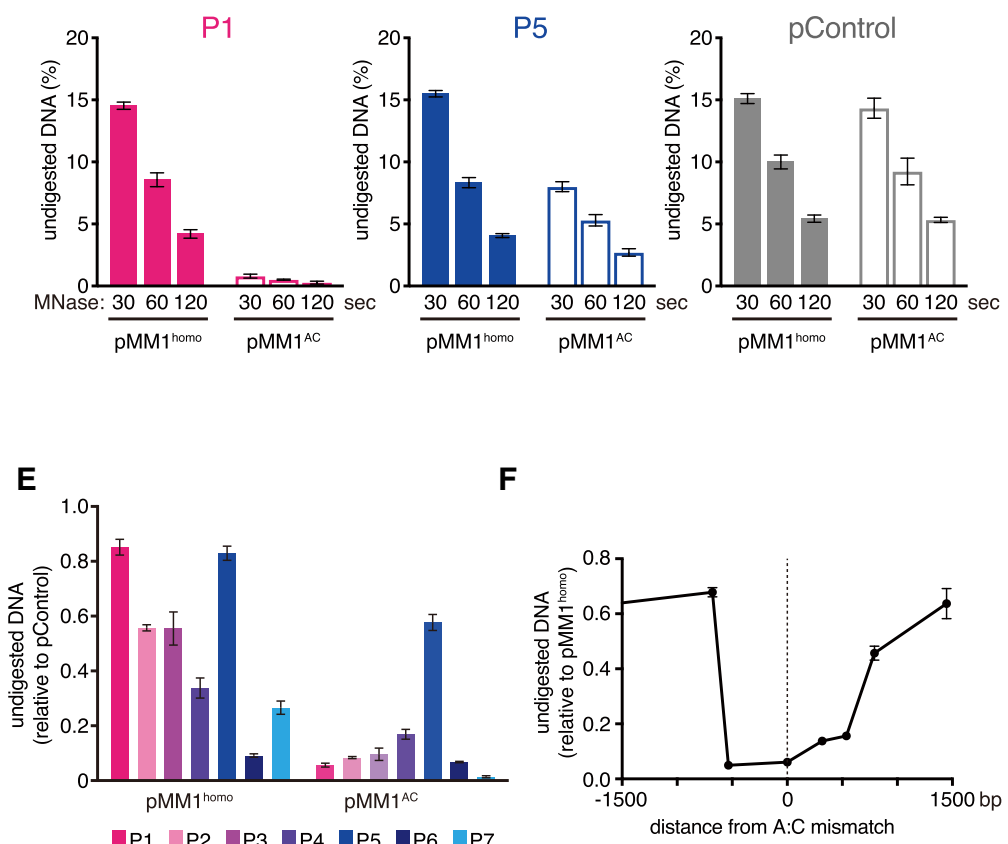

$\mathbf{F}$



Figure 1. Nucleosomes are excluded from a $>1-\mathrm{kb}$ region surrounding a mismatch. $(A)$ The DNA substrate used in this study. The 3011 base-pair (bp) DNA carries an A:T base pair (pMM1 ${ }^{\text {homo }}$ ) or an A:C mispair (pMM1 ${ }^{\mathrm{AC}}$ ) at position 1. Positions of restriction enzyme sites used in this study, the site of biotin modification, and amplicons for quantitative PCR (qPCR) (P1: 2950-61, P2: 253-383, P3: 476-602, P4: 728-860, P5: 1498-1628, P6: 2266-2397, and P7: 2413-2537) are indicated. (B) Supercoiling assay in NPE. Covalently closed pMM1 homo (lanes 2-8) or pMM1 ${ }^{\text {AC }}$ (lanes 9-15) was incubated in NPE and sampled at the indicated times. (Lane 1) Supercoiled pMM1 ${ }^{\text {homo }}$ purified from Escherichia coli was used as a size standard. (oc/r) Open circular or relaxed DNA; (sc) supercoiled DNA. (C) pMM1 ${ }^{\text {homo }}($ lanes $1-4)$ or $\mathrm{pMM1}^{\mathrm{AC}}$ (lanes 5-8) was incubated in NPE for $60 \mathrm{~min}$ and digested by micrococcal nuclease (MNase). DNA samples stained with SYBR Gold (top) and Southern blotting with the PvuII-PvuII probe (middle) and the DraI-DraI probe (bottom) are shown. $(D-F)$ The MNase assay described in $C$ was repeated in the presence of a control plasmid (pControl), and undigested DNA was quantified by qPCR. The amount of DNA relative to the input $(D)$ and normalized to pControl $(E)$ and $\mathrm{pMM1}{ }^{\text {homo }}(F)$ is presented. Mean \pm one standard deviation $(\mathrm{SD})$ is shown. $n=3$.

3 min (Fig. 1B, lanes 2-6). Since NPE recapitulates the S-phase nuclear environment that does not allow prereplicative complex assembly, no DNA replication initiates when a plasmid is directly incubated in NPE (Walter et al. 1998). Therefore, chromatin assembly in this experiment was mediated mostly by the HIRA-dependent pathway (see Supplemental Fig. S5A,B).

A single-strand break or DNA-bound PCNA can induce strand-specific MMR in vitro (Holmes et al. 1990; Thomas et al. 1991; Pluciennik et al. 2010; Kawasoe et al. 2016). If neither feature is present, MutS $\alpha$ or MutS $\beta$ binds to mismatches and translocates along DNA as a sliding clamp, but MutLa-dependent strand incision does not occur. Since the signal search reaction should require remote communication between a mismatch and strand signal, we wondered whether this step is associated with any alterations in chromatin structure. Interestingly, we found that a plasmid carrying an $\mathrm{A}: \mathrm{C}$ mismatch $\left(\mathrm{pMM}{ }^{\mathrm{AC}}\right.$ ) (Fig. 1A) is not significantly supercoiled in NPE (Fig. 1B). Other base-base mismatches and a single IDL also 
inhibited supercoiling of a plasmid (Supplemental Fig. S1A). To see whether the inhibition of supercoiling is due to a reduction of the number of nucleosomes on DNA, we digested the plasmid with micrococcal nuclease (MNase). Southern blotting of the DNA showed that the mismatch-proximal region was highly sensitive to MNase (Fig. 1C). To quantitatively map the region where the MNase sensitivity is increased, we repeated the MNase digestion assay in the presence of an unrelated "control" plasmid (pControl) and quantified undigested DNA fragments by quantitative PCR (qPCR). This assay confirmed that the mismatch-proximal region $(\mathrm{P} 1)$ is highly susceptible to MNase, and even the most mismatch-distal region (P5), which is $\sim 1.5 \mathrm{~kb}$ away from the mismatch, is weakly affected (Fig. 1D; Supplemental Fig. S1B). The MNase sensitivity of the control plasmid was not detectably changed by the coincubation of pMM1 ${ }^{\mathrm{AC}}$ (Fig. 1D, "pControl"), indicating that the increase of the MNase sensitivity occurs in cis. We also found that the relative MNase sensitivity is most strongly enhanced within an $\sim 1-\mathrm{kb}$ region surrounding the mismatch (Fig. 1E,F; Supplemental Fig. S1C-E). Here, we refer to this reaction as nucleosome exclusion.

\section{Nucleosome exclusion depends on the Msh2- containing complexes and involves nucleosome disassembly}

To test whether nucleosome exclusion depends on the Msh2-dependent MMR system, we immunodepleted both MutS $\alpha$ and MutS $\beta$ from NPE (Fig. 2A). As shown in Figure 2B, depletion of the Msh2-containing complexes relieved the inhibition of supercoiling on the mismatch-carrying plasmid. Depletion of Msh6 was sufficient to both allow supercoiling of the mismatch-carrying plasmid and inhibit gap-directed MMR of a base-base mismatch (Supplemental Fig. S2A-D). Depletion of NPE with four different Msh2 or Msh6 antibodies consistently allowed supercoiling of the mismatch-carrying plasmid (Supplemental Fig. $\mathrm{S} 2 \mathrm{~A}, \mathrm{~B})$, strongly suggesting that the MutSa complex is primarily responsible for nucleosome exclusion around a base-base mismatch. However, the mismatch-dependent inhibition of supercoiling was not efficiently restored by the addition of recombinant MutSa to Msh2-depleted NPE (Fig. 2B). The reason for the failure of the rescue is currently not clear, but unidentified factors required for nucleosome exclusion might be codepleted with MutSa. It should also be noted that recombinant MutSa can restore gap-directed MMR in NPE (Kawasoe et al. 2016), suggesting that the level of nucleosome exclusion that is detectable in the supercoiling assay is dispensable for the repair of mismatches at least in the gap-directed system with naked DNA substrates (see below). Msh3 is $>100$-fold less concentrated than Msh2 in Xenopus egg extracts (Supplemental Fig. S2E), and, possibly because of its low concentration, the effect of MutS $\beta$ depletion on nucleosome exclusion was not visible in our experiments (Supplemental Fig. S2F,G). Depletion of Mlh1, which abolished gap-directed MMR (Supplemental Fig. S2H), did not inhibit nucleosome exclusion (Fig. 2C,D), suggesting that the
Mlh1-containing complexes (Mlh1-Pms2, Mlh1-Pms1, and Mlh1-Mlh3) are dispensable for nucleosome exclusion. From these data, we infer that an Msh2-dependent but Mlh1-independent reaction facilitates nucleosome exclusion.

We also tested whether the exclusion reaction involves the displacement of preassembled nucleosomes (Fig. 2E). To enhance nucleosome exclusion, we used a $3.0-\mathrm{kb}$ plasmid carrying three mismatches. The mismatch-carrying plasmid was fully supercoiled in an Msh2-depleted NPE (Fig. 2F, lane 10; see Supplemental Fig. S2I for Msh2 depletion). However, upon transfer into the second Msh2-containing NPE, plasmids with relative linking numbers of less than -6 were detectably decreased (Fig. $2 \mathrm{~F}$, cf. lanes 11 and 12). The displacement of nucleosomes likely occurred around the site of mismatches, since the MNase sensitivity was increased preferentially around mismatch sites (Fig. 2G). These data suggest that nucleosome exclusion is associated with active disassembly of nucleosomes.

\section{Chromatin remodeling enzyme Smarcad1 is recruited to mismatch-carrying DNA}

To clarify the mechanism of nucleosome exclusion, we looked for factors that are recruited to mismatch-carrying DNA by Msh2-containing complexes. We immobilized plasmid DNA on Sepharose beads through a site-specific biotin modification (see Fig. 1A), incubated them in NPE, recovered the plasmid DNA, and compared the relative abundance of chromatin-binding factors by mass spectrometry (Fig. 3A-C). As expected, peptides corresponding to Msh2, Msh6, and Mlh1 were found preferentially on the mismatch DNA (Fig. 3C). The spectral counts of known chromatin-related factors such as HIRA and Smarca5 (ISWI) were reduced in the presence of a mismatch, probably because DNA was less chromatinized. However, the spectral counts of Smarcad1 and the FACT subunits Spt16 and Ssrp1 were increased in the presence of a mismatch.

To quantitatively compare chromatin binding of these factors, we repeated the plasmid pull-down assay and blotted each factor with specific antibodies (Fig. 4A-C; Supplemental Fig. S3). Loading of Histones H2B, H3, and H4 was significantly reduced in the presence of a mismatch, and this effect was dependent on Msh2 but not Mlh1 (Fig. 4A-C). Smarcad1 was specifically recruited onto the mismatch-carrying DNA (Fig. 4B [lanes 1,2], C). Critically, mismatch-specific loading of Smarcad1 was dependent on Msh2 but not Mlh1 (Fig. 4B [lanes 2,4,6], C). Consistent with the mass spectrometry data, Spt16 and Ssrp1 were found on DNA in the absence of a mismatch. Chromatin binding of FACT subunits may be increased in the presence of a mismatch, but the difference was not statistically significant with our sample number $(n=$ 4) (Fig. 4C). By immunoprecipitation, a small amount of Smarcad1 was coprecipitated with Msh2 and Msh6, and Smarcad1 coprecipitated Msh2 and Msh6 (Fig. 4D,E), suggesting that Smarcad1 physically interacts with MutSa, as reported in human cells (Okazaki et al. 2008; Rowbotham et al. 2011; Chen et al. 2016). These results identify 
A

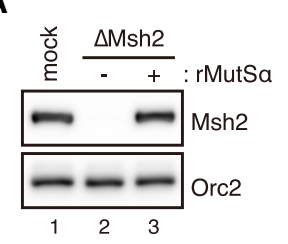

C



B
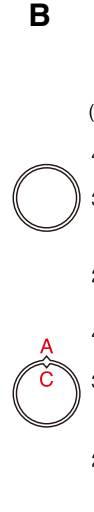
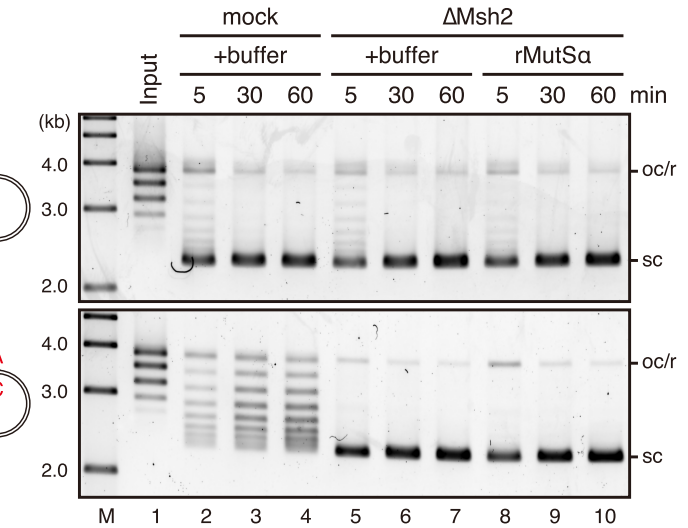

D

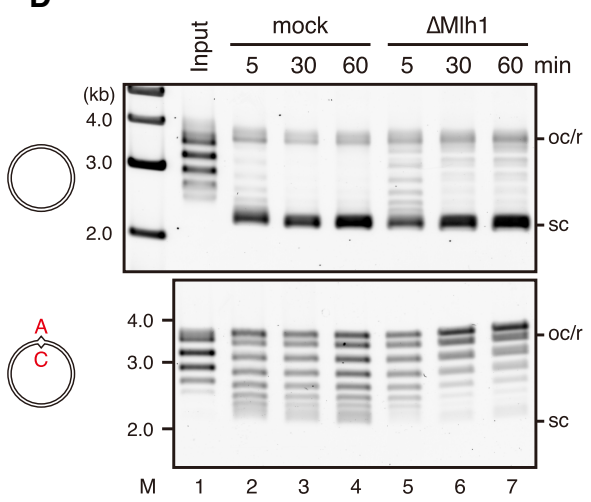
$E$ Sepharose

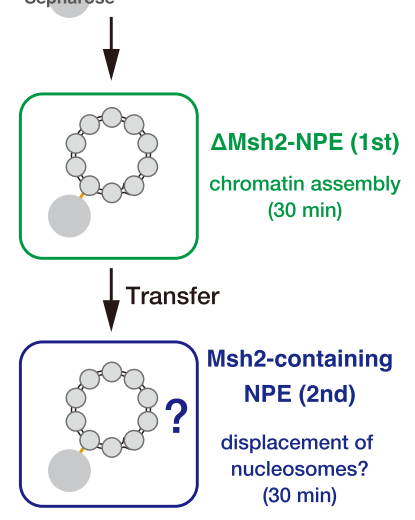

G



$\mathbf{F}$

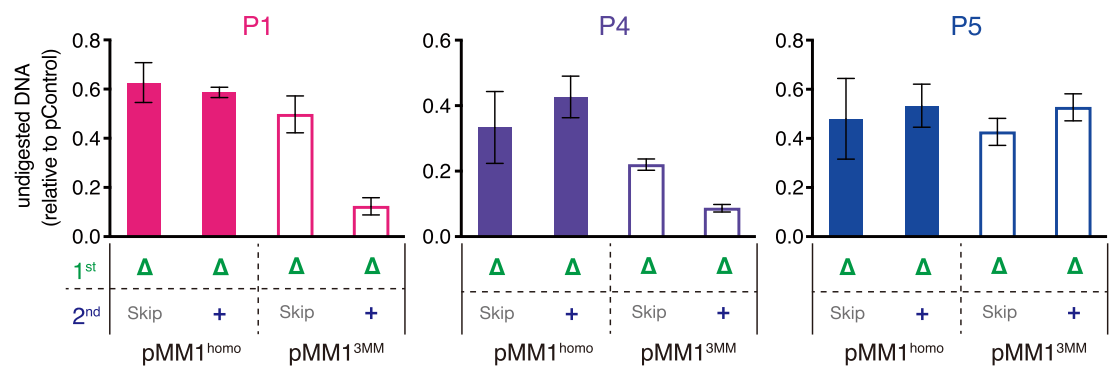

Figure 2. Nucleosome exclusion depends on the Msh2-containing complexes and involves nucleosome disassembly. (A) The immunodepletion efficiency of Msh2. To enhance the depletion efficiency, a mixture of Msh2 and Msh6 antibodies was used. Mock-treated (lane 1, mock) or Msh2-depleted (and Msh6-depleted) NPE (lanes 2,3, $\Delta$ Msh2) (0.25 $\mu \mathrm{L}$ of each) supplemented with either buffer (lanes 1,2) or 900 $\mathrm{nM}$ recombinant MutSa (lane 3) was separated by SDS-PAGE and probed with the indicated antibodies. Orc2 served as a loading control. The depletion efficiency was estimated as $99 \%$. See also Figure 4A for codepletion of Msh6, Msh3, and other factors. (B) Supercoiling assay with pMM1 ${ }^{\text {homo }}(t o p)$ or $\mathrm{pMM1}^{\mathrm{AC}}$ (bottom) in NPE as described in A. See Supplemental Figure S2A-D for supercoiling and gap-directed MMR in Msh2- or Msh6-depleted NPE. (C) The immunodepletion efficiency of Mlh1. The depletion efficiency was estimated as $98 \%$. $(D)$ Supercoiling assay in NPE as described in C. See Supplemental Figure S2H for gap-directed MMR. (E) Schematic diagram of the nucleosome displacement assay. pMM1 carrying three mismatches at positions 1 (A:C), 803 (A:C), and 2271 (T:C) (pMM1 ${ }^{3 M M}$; see also $G)$ was immobilized on Sepharose beads and incubated in an Msh2-depleted NPE for $30 \mathrm{~min}$. The plasmid was then transferred into the second NPE containing Msh2, incubated for an additional $30 \mathrm{~min}$, and recovered. $(F)$ Nucleosome displacement assay. pMM1 ${ }^{\text {homo }}$ (lanes 1-6) or $\mathrm{pMM1}^{3 \mathrm{MM}}$ (lanes 7-12) was sequentially incubated in the indicated extracts. $(+)$ Mock-treated NPE; $(\Delta)$ Msh2-depleted NPE; (Skip) no incubation. The linking number of each band relative to the open circular or relaxed DNA (oc/r) position $(\Delta \mathrm{L})$ is indicated at the right of the gel. The ratio of the plasmids of the indicated $\Delta \mathrm{L}$ was quantified and is presented as a graph. Mean \pm one $\mathrm{SD}$ is shown. $n=$ 5. $(G)$ The nucleosome displacement assay was repeated without plasmid immobilization and in the presence of pControl. Instead of transferring plasmids, the second NPE was added directly to the first NPE to supply Msh2. The amount of DNA fragments relative to pControl after $60 \mathrm{sec}$ of MNase digestion was quantified by qPCR. Mean \pm one SD is shown. $n=3$. 
A

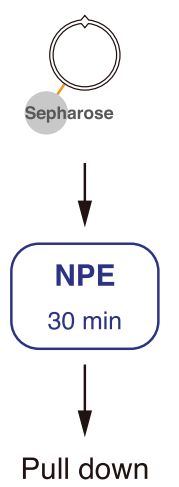

B



C

\begin{tabular}{l|c|c|c|c|c}
\hline \multirow{2}{*}{ Protein } & \multirow{2}{*}{$\boldsymbol{M}_{\boldsymbol{r}}(\mathbf{K})$} & \multicolumn{3}{|c}{ Spectral counts } \\
\cline { 3 - 6 } & & \multicolumn{2}{|c|}{ Ex \#1 } & \multicolumn{2}{|c}{ Ex \#2 } \\
\cline { 3 - 6 } & \multirow{2}{*}{ A:T } & A:C & A:T & A:C \\
\hline Msh6 & 150 & 0 & 202 & 7 & 157 \\
Msh2 & 104 & 0 & 100 & 12 & 102 \\
Mlh1 & 84 & 1 & 25 & 13 & 18 \\
Smarcad1 & 115 & 0 & 12 & 0 & 9 \\
Spt16 & 118 & 44 & 78 & 35 & 68 \\
Ssrp1 & 79 & 20 & 27 & 13 & 30 \\
HIRA & 121 & 28 & 14 & 8 & 3 \\
Smarca5 & 122 & 19 & 7 & 28 & 13 \\
\hline
\end{tabular}

Figure 3. Identification of factors that are recruited onto mismatch-carrying DNA. (A) Schematic diagram of the plasmid pull-down assay. (B) Silver staining of mismatch DNA-binding factors. Samples were separated by SDS-PAGE and stained with silver nitrate. (A:T) pMM1 ${ }^{\text {homo }}$ ( $\left.\mathrm{A}: \mathrm{C}\right) \mathrm{pMM1}{ }^{\mathrm{AC}}$. " $\mathrm{M}$ " indicates size markers. $(C)$ Summary of mass spectrometry analysis. Spectral counts of the indicated proteins are listed along with their molecular masses. (Ex \#1 and Ex \#2) Independent experiments. See also Supplemental Table S1 for the complete list of identified factors.
Smarcad1 as a factor that is recruited onto mismatch-carrying DNA by an Msh2-dependent mechanism. Since FACT showed very high nonspecific binding to control IgG beads, we were not able to perform reliable experiments on the interaction between FACT and MutSa (Fig. 4D).

\section{Smarcad1 promotes mismatch-dependent exclusion of nucleosomes}

We tested whether Smarcad1 promotes nucleosome exclusion. In an NPE depleted of $\sim 98 \%$ Smarcad1, we did not see a detectable change in supercoiling in the absence of a mismatch, suggesting that Smarcad1 does not play a major role in nucleosome assembly in this system (Fig. 5A,B; see Supplemental Fig. S4A for Smarcad1 depletion). In the presence of a mismatch, however, plasmids that have relative linking numbers of less than -3 were accumulated, and this accumulation was reverted by the addition of wild-type but not the Walker A mutant Smarcad1 (Fig. 5B; Supplemental Fig. S4F, G [see B for recombinant Smarcad1]). We also found that regions surrounding the mismatch become more resistant to MNase in the absence of Smarcad1, an effect that is reversed by recombinant Smarcad1 (Fig. 5C; Supplemental Fig. S4C-E). These results suggest that Smarcadl functions as an ATPase to facilitate nucleosome exclusion.

As the effect of Smarcad1 depletion was partial compared with Msh2 depletion, we included FACT in our supercoiling assay to see whether FACT also contributes to nucleosome exclusion (Fig. 5D,E). Although depletion of FACT by itself had no detectable effect, simultaneous depletion of Smarcad1 and FACT further enhanced supercoiling of the plasmid in the presence of a mismatch (Fig. 5D,E; Supplemental Fig. S4H,I). These data suggest that FACT also assists nucleosome exclusion, albeit to a lesser extent.

\section{Nucleosome exclusion counteracts synthesis-coupled chromatin assembly}

Since MMR is a post-replicative repair system, CAF-1-mediated chromatin assembly may be more relevant to
MMR. NPE efficiently converts a primed single-stranded plasmid to the double-stranded form (Fig. 6A). Because unregulated priming is suppressed in NPE (Walter and Newport 2000), DNA synthesis initiates from the $3^{\prime}$ terminus of the primer, and the MMR system can use either terminus of the primer as a strand discrimination signal. In this system, as expected, supercoiling of the primer extension products depended on both HIRA and CAF-1, while that of double-stranded plasmids depended only on HIRA (Supplemental Fig. S5A,B). A mismatch on the primer was efficiently repaired, and the repair was partially dependent on Msh2 and Mlh1 (Fig. 6B; Supplemental Fig. S5C). The mismatch correction seen in Msh2- and Mlh1-depleted NPE may be mediated by proofreading by DNA polymerases or flap processing during the completion of synthesis. Importantly, in the absence of Mlh1, DNA products that escaped from Mlh1-independent mismatch correction were not supercoiled (Fig. 6C, lane 11; Supplemental Fig. S5D), and the inhibition of supercoiling was Msh2-dependent (Fig. 6C, lane 13). Since depletion of either HIRA or CAF1 was insufficient for preventing supercoiling of the primer extension products, we infer that nucleosome exclusion can counteract both HIRA- and CAF-1-mediated chromatin assembly.

To test how Smarcad1 contributes to the inhibition of supercoiling in this system, we combined depletion of Smarcad1 with that of Mlh1 and HIRA. Indeed, in the absence of Smarcad1, regardless of the presence or absence of HIRA, the plasmids with relative linking numbers of less than -6 were significantly accumulated, and the effect was reversed by recombinant Smarcad1 (Fig. 6D,E; Supplemental Fig. S5E-H). These data are in good agreement with the hypothesis that Smarcad1 assists Msh2-dependent nucleosome exclusion to counteract both HIRAand CAF-1-mediated chromatin assembly.

\section{Smarcad1 facilitates the repair of mismatches when $D N A$ is chromatinized}

An important question is whether Smarcad1 and FACT promote replication error correction in the context of chromatin. Because it is not possible at this point to quantitatively measure replication-coupled error correction in 
Terui et al.

A

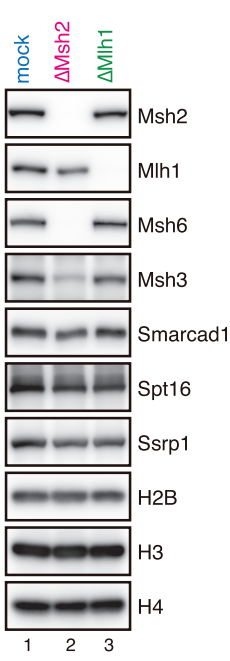

B

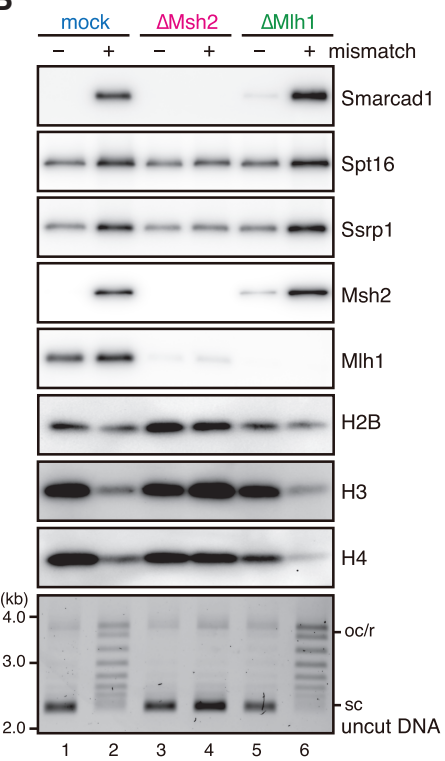

D

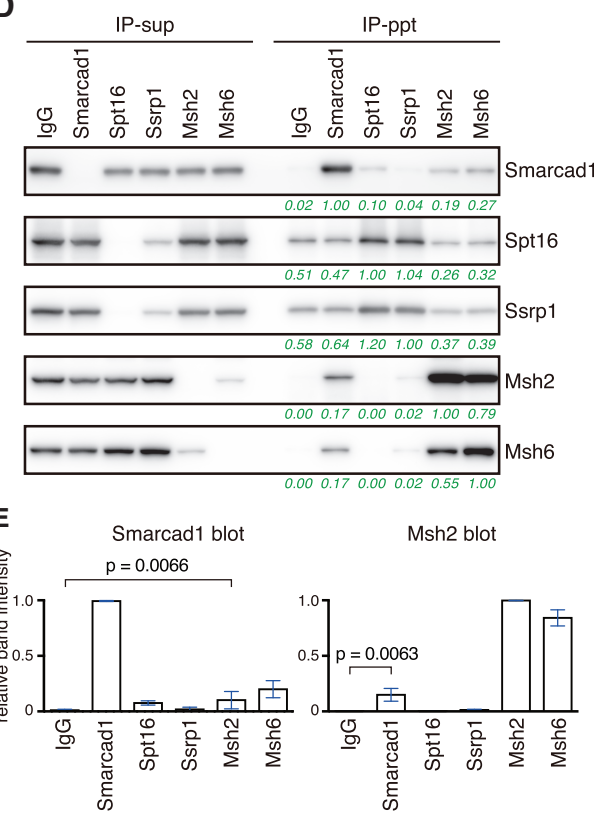

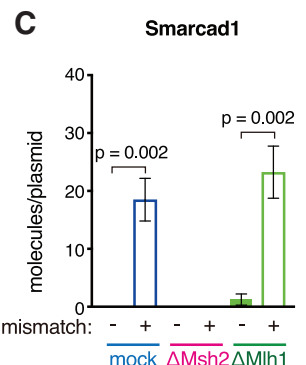

Mih1

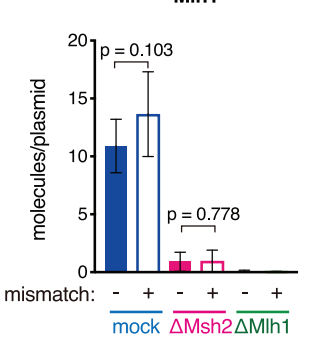

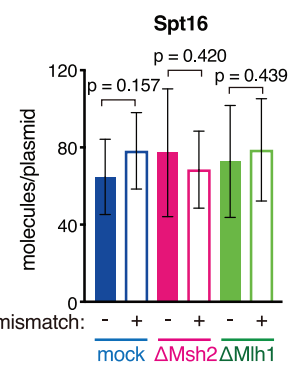

H2B

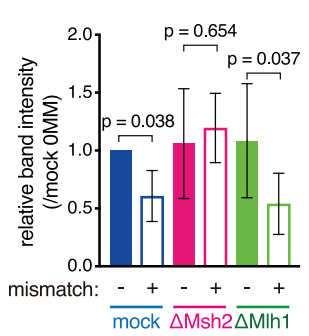

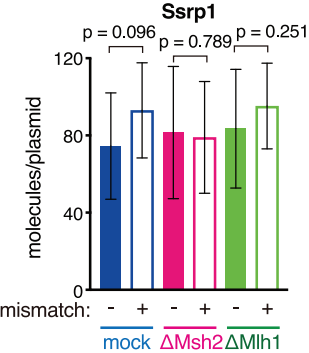

H3

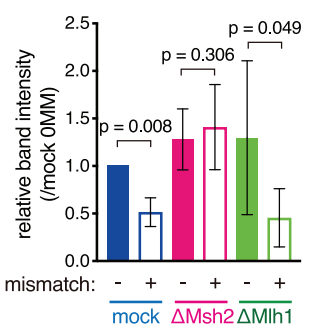

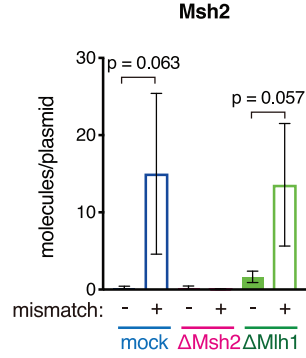

H4

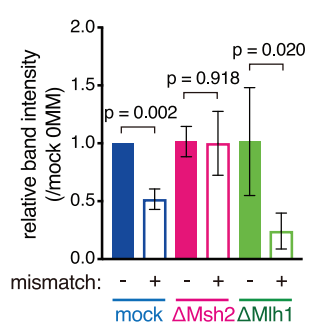

Figure 4. Smarcad1 is recruited onto mismatch-carrying DNA in a Msh2-dependent manner. $(A)$ The immunodepletion efficiencies of Msh2 or Mlh1. NPE was depleted using preimmune antibodies (lane 1, mock), a mixture of Msh2 and Msh6 antibodies (lane 2, $\Delta$ Msh2), or Mlh1 antibodies (lane 3, $\Delta$ Mlh1). NPE $(0.25 \mu \mathrm{L})$ was separated by SDS-PAGE and probed with the indicated antibodies. The depletion efficiencies for Msh2 and Mlh1 were estimated as $99 \%$ and $98 \%$, respectively. $(B)$ Immobilized pMM1 ${ }^{\text {homo }}($ lanes $1,3,5)$ or pMM1 ${ }^{\text {AC }}($ lanes $2,4,6)$ was incubated in NPE as described in $A$ and recovered. Immunoblotting of the indicated antibodies and uncut DNA stained with SYBR Gold is presented. (C) Quantification of chromatin-binding factors. Band intensities were normalized to the amount of DNA quantified by qPCR. For Smarcad1, Msh2, Mlh1, Spt16, and Ssrp1, the number of molecules on a plasmid was estimated by using recombinant proteins as standards. Histones were normalized to the amount on no mismatch DNA in the mock sample. Mean \pm one SD is shown. $n=4$. $P$-values were calculated by the paired $t$-test (two-tailed). $(D)$ Coimmunoprecipitation of Smarcadl and MutSa. Immunoblotting of supernatant (IP-sup) and bead (IP-ppt) samples is presented. Green numbers represent band intensities relative to the target protein of immunoprecipitation. (E) Quantification of immunoprecipitated proteins. Mean \pm one SD is shown. $n=3 . P$-values were calculated by the paired $t$-test (two-tailed).

Xenopus egg extracts, we undertook three approaches. First, we used the gap-directed MMR assay. However, we observed no reproducible reduction in the MMR efficiencies by depletion of Smarcad1, FACT, or both even when the mismatch gap distance was extended to $1.9 \mathrm{~kb}$ (Supplemental Fig. S6A,B). Second, we used the primer extension-based MMR assay. Again, we did not see a detectable reduction of the MMR efficiencies by depletion of 
A

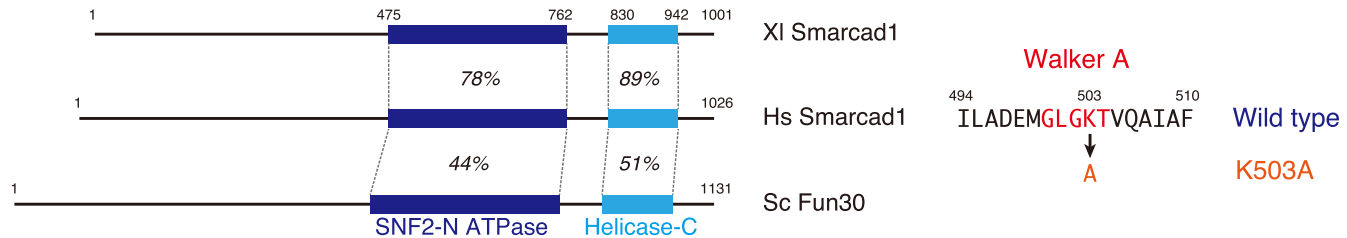

B

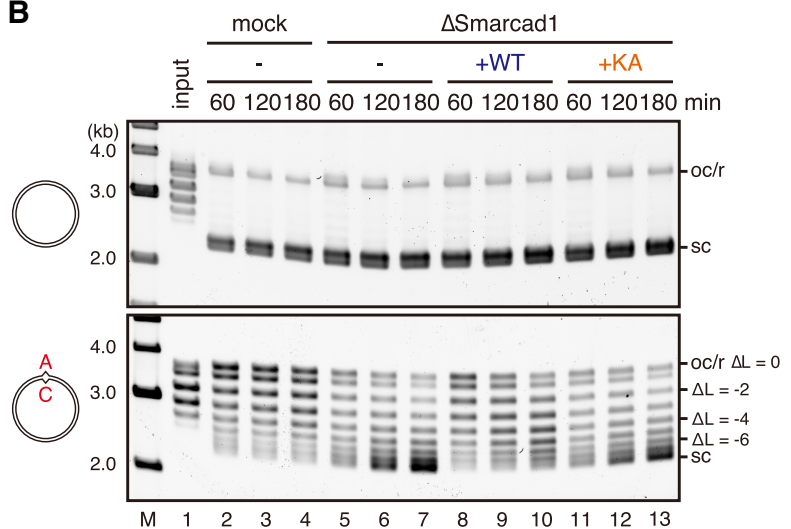

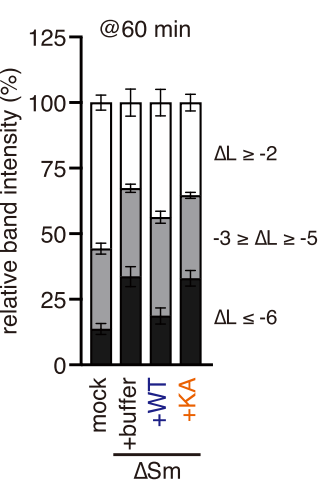

$\mathbf{C}$

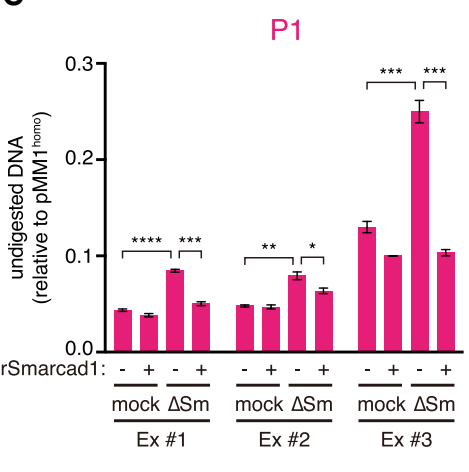

D

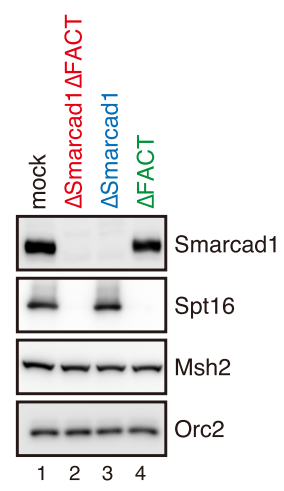

E

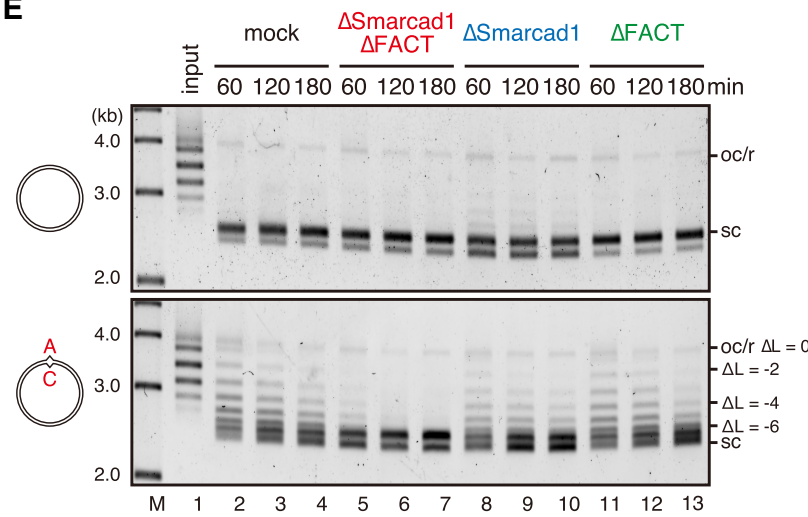

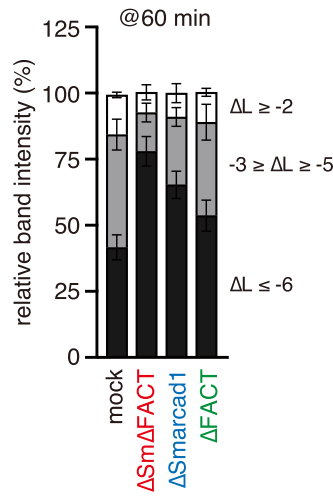

Figure 5. Smarcadl and FACT assist nucleosome exclusion. (A) The domain architecture of Xenopus laevis (Xl) and Homo sapiens (Hs) Smarcad1 and Saccharomyces cerevisiae (Sc) Fun30. The positions and sequence identities of the SNF2 family N-terminal domain (SNF2$\mathrm{N}$ ATPase) and helicase C-terminal domain (Helicase-C) are indicated. The sequence of isoform A was used for Xl Smarcad1. The amino acid sequence of the Walker A motif is presented. (B) Supercoiling assay in Smarcad1-depleted NPE. The linking number of each band relative to the open circular or relaxed DNA $(\mathrm{oc} / \mathrm{r})$ position $(\Delta \mathrm{L})$ is indicated. The ratio of the plasmids of the indicated $\Delta \mathrm{L}$ was quantified and is presented as a graph. Mean \pm one SD is shown. $n=3$. See Supplemental Figure S4A for depletion efficiencies. $(C)$ The MNase assay was performed as described in Figure 1D using Smarcad1-depleted NPE. (Ex \#1, Ex \#2, and Ex \#3) Independent experiments. The amount of undigested DNA relative to $\mathrm{pMM} 1^{\text {homo }}$ is plotted as a graph. Mean \pm one SD is shown. $n=3$ technical replicates. $P$-values were calculated

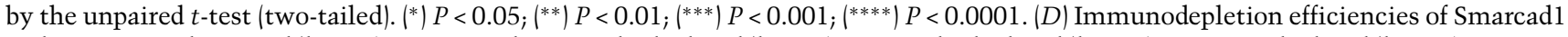
and FACT. Mock-treated (lane 1), FACT- and Smarcad1-depleted (lane 2), Smarcad1-depleted (lane 3), or FACT-depleted (lane 4) NPE was separated by SDS-PAGE and probed with the indicated antibodies. NPE $(0.25 \mu \mathrm{L}$ of each) was loaded. The depletion efficiencies for Smarcad 1 and Spt 16 were estimated as $98 \%$ and $95 \%$, respectively. $(E)$ The supercoiling assay in NPE described in $D$. The ratio of the plasmids of the indicated $\Delta \mathrm{L}$ was quantified and is presented as a graph. Mean \pm one SD is shown. $n=3$.

Smarcad1 (Supplemental Fig. S6C-E). In both approaches, we started the reaction by adding naked DNA substrates into NPE. If the recognition of mismatches is significantly quicker than chromatin assembly, these approaches may not be appropriate for testing the effect of chromatin on MMR. Third, we set up a system where MMR is initiated on a chromatinized template. We preassembled nucleosomes on a gap-carrying DNA in the absence of Msh2 and Smarcad1 and then supplied Msh2 to initiate the MMR reaction (Fig. 7A). Since the strand discrimination signal (a gap) is quickly filled in NPE, we repressed gap filling by inhibiting the PCNA function with a PCNA- 
A

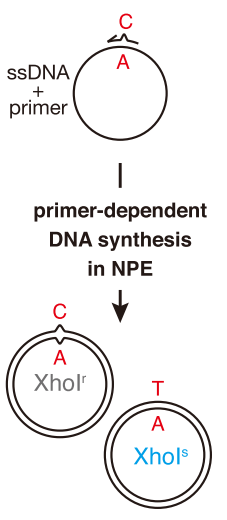

D
B

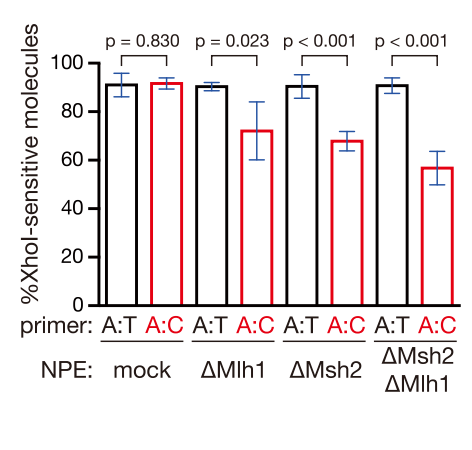

nuclease treated

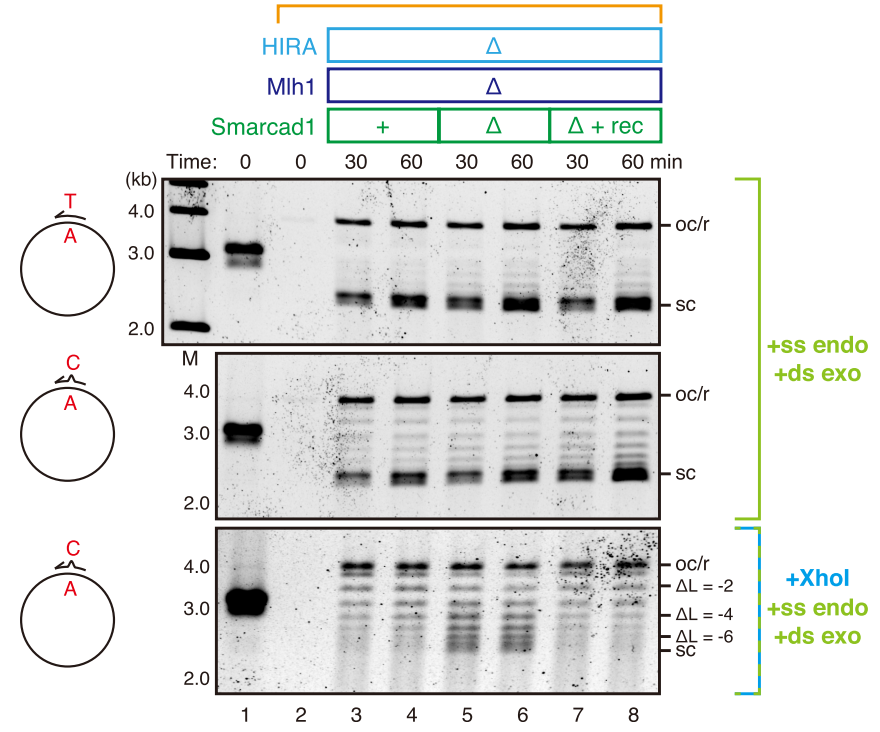

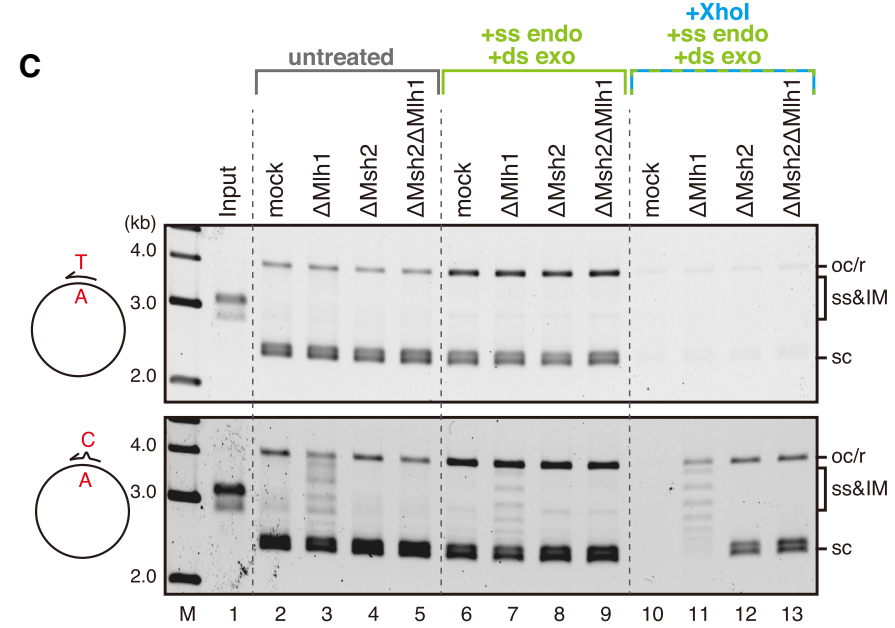

E

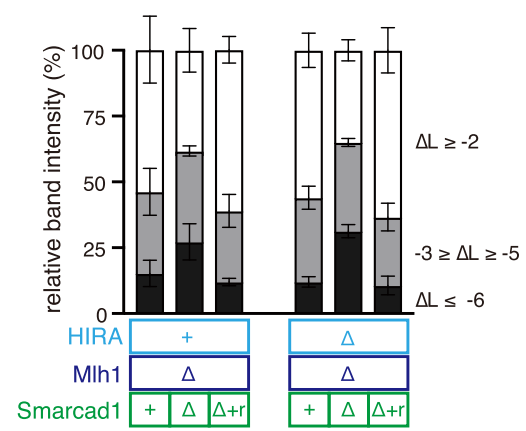

Figure 6. The nucleosome exclusion reaction counteracts DNA synthesis-coupled chromatin assembly. $(A)$ Schematic diagram of the primer extension assay. A 92-nucleotide (nt) primer carrying either no mismatch or an A:C mismatch is annealed on a single-stranded pMM1. Upon incubation in NPE, complementary DNA is synthesized depending on the primer, converting the substrate into covalently closed circular DNA. (B) The requirements of canonical MMR factors for primer extension-coupled mismatch correction. The primer extension assay was performed in mock-treated, Mlh1-depleted ( $\Delta$ Mlh1), Msh2-depleted ( $\Delta$ Msh2), or Msh2/Mlh1 doubly depleted $(\Delta \mathrm{Msh} 2 \Delta \mathrm{Mlh} 1) \mathrm{NPE}$. The ratio of XhoI-sensitive molecules that correspond to the C-to-T repair products is plotted in a graph. Mean \pm one SD is shown. $n=4$. $P$-values were calculated by the unpaired $t$-test (two-tailed). Note that the ratio did not reach $100 \%$ even with a homoduplex primer because of the presence of some residual primer extension intermediates. See also Supplemental Figure S6, D and $\mathrm{E}$, for the details of quantification. (C) Nucleosome exclusion on the primer extension products. The products described in $B$ were separated by agarose gel without any treatment (lanes 2-5), after digestion of incomplete intermediates by S1 nuclease and ExoV (lanes 6-9), or after digestion of C-to-T repair products and incomplete intermediates by XhoI, S1 nuclease, and $\lambda$ exonuclease (lanes 10-13). (ss) ssDNA; (IM) primer extension intermediates. $(D)$ The assay presented in $C$ was repeated in NPE depleted of Mlh1 and HIRA (lanes 3,4) or Mlh1, HIRA, and Smarcad1 (lanes 5-8) supplemented with either buffer (lanes 3-6) or recombinant Smarcad1 (lanes 7,8). The linking number of each band relative to the open circular or relaxed DNA $(\mathrm{oc} / \mathrm{r})$ position $(\Delta \mathrm{L})$ is indicated at the right of the gel. $(E)$ The ratio of the plasmids of the indicated $\Delta \mathrm{L}$ in $D$ and Supplemental Figure S5F was quantified and is presented as a graph. Mean \pm one SD is shown. $n=3$.

binding peptide derived from p21 (Supplemental Fig. S6F, G; Kawasoe et al. 2016). Stepwise incubation did not significantly reduce the MMR efficiency when the gap was retained by the p21 peptide (Supplemental Fig. S6G, cf. lanes 1-4 and 13-16). Interestingly, however, we observed a statistically significant reduction of the MMR efficiency in the absence of Smarcad1, and the effect was restored by recombinant Smarcad1 (Fig. 7B-E). These results suggest that Smarcad1 facilitates Msh2-dependent MMR when nucleosomes are assembled around a mismatch. 
A

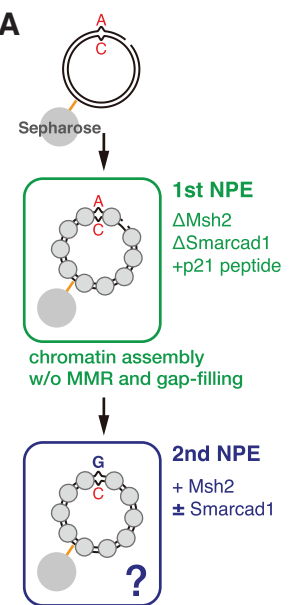

MMR in the presence of pre-assembled nucleosomes?

D



B



C


Figure 7. Smarcadl facilitates MMR in the presence of preassembled nucleosomes. (A) Schematic diagram of the stepwise incubation assay. $\mathrm{pMM1}^{\mathrm{AC}}$ carrying a 15-nt gap on the A strand was immobilized on Sepharose beads and incubated in an Msh2- and Smarcad1depleted NPE (1st NPE) containing $1 \mathrm{mg} / \mathrm{mL}$ p21 PCNA-binding peptide ( $\mathrm{NH}_{2}$-KRRQTSMTDFYHSKRRLIFS-COOH) for $30 \mathrm{~min}$. The plasmid was then transferred into the second NPE (2nd NPE) containing Msh2 and incubated for the indicated times. $(B)$ The immunodepletion efficiencies of MutSa and Smarcad1. $\left(^{*}\right)$ Cross-reacting band. (C) Supercoiling assay in the first NPE. See also Supplemental Figure S6, F and G, for the effect of the p21 peptide on gap retention. $(D)$ MMR efficiencies after the incubation in the second NPE. DNA was digested with XmnI and either BamHI (A-to-G repair; top) or XhoI(C-to-T repair; bottom). The percentage of repair was calculated based on the percentage of XhoI- or BamHI-sensitive DNA molecules. $(E)$ Statistical analysis of the effect of Smarcad1 on MMR in the stepwise incubation assay. The A-to-G repair efficiencies are plotted in a graph. Mean \pm one $S D$ is shown. $n=3$. $P$-values were calculated by the paired $t$-test (two-tailed). Blue triangles indicate individual values.

The yeast homolog of Smarcad1 is an accessory factor for Msh2-dependent MMR

To assess the contribution of the Smarcad1 homolog to replication error correction in the context of chromatin replication, we used the budding yeast Saccharomyces cerevisiae. Homopolymer runs of adenine or thymine are known hot spots for slippage of DNA polymerases, and MMR very efficiently corrects such slippage errors (Kunkel and Erie 2015). In this study, we measured the reversion of hom3-10 and lys2::insE-A14, both of which detect -1 frameshifts in $\mathrm{A} / \mathrm{T}$ runs (Marsischky et al. 1996; Tran et al. 1997).
The budding yeast genome encodes one Smarcad1 homolog, Fun30 (see Fig. 5A). fun $30 \Delta$ increased the reversion rate by 2.1 -fold $(P$-value $=0.0024$, wild type vs. fun $30 \Delta)$ at hom3 and by 1.9-fold $(P$-value $<0.0001)$ at lys2, suggesting a possibility that Fun 30 contributes to the suppression of spontaneous mutations (Table 1). To test the possibility that Fun30 is an accessory factor for the MMR system, we evaluated genetic interactions between Fun30 and MMR factors by making double mutants. In budding yeast, due to their functional overlap, either $m s h 6 \Delta$ ( $\Delta$ MutSa) or msh3 $\Delta$ ( $\Delta$ MutS $\beta$ ) causes only a partial increase of the mutation rates for these frameshift assays (Marsischky et al. 1996). Interestingly, when FUN30 was disrupted 
Table 1. Reversion rates at the hom3-10 and lys2::insE-A14 loci

\begin{tabular}{|c|c|c|c|c|}
\hline \multirow[b]{2}{*}{ Genotype } & \multicolumn{2}{|l|}{ hom3-10 } & \multicolumn{2}{|l|}{ lys2-14A } \\
\hline & Reversion rate & Fold increase & Reversion rate & Fold increase \\
\hline Wild type & $1.6 \times 10^{-9}\left(8.8 \times 10^{-10}-2.4 \times 10^{-9}\right)$ & 1.0 & $3.7 \times 10^{-8}\left(3.0 \times 10^{-8}-4.6 \times 10^{-8}\right)$ & 1.0 \\
\hline fun30s & $3.3 \times 10^{-9}\left(2.2 \times 10^{-9}-4.7 \times 10^{-9}\right)$ & 2.1 & $7.0 \times 10^{-8}\left(5.8 \times 10^{-8}-8.2 \times 10^{-8}\right)$ & 1.9 \\
\hline fun30-K603A & $5.1 \times 10^{-9}\left(3.3 \times 10^{-9}-7.2 \times 10^{-9}\right)$ & 3.3 & $9.3 \times 10^{-8}\left(7.7 \times 10^{-8}-1.1 \times 10^{-7}\right)$ & 2.5 \\
\hline msh64 & $1.0 \times 10^{-8}\left(7.0 \times 10^{-9}-1.4 \times 10^{-8}\right)$ & 6.5 & $6.9 \times 10^{-6}\left(5.6 \times 10^{-6}-8.4 \times 10^{-6}\right)$ & 190 \\
\hline$m s h 6 \Delta$ fun30 & $1.2 \times 10^{-7}\left(1.0 \times 10^{-7}-1.5 \times 10^{-7}\right)$ & 79 & $4.0 \times 10^{-5}\left(3.5 \times 10^{-5}-4.5 \times 10^{-5}\right)$ & 1100 \\
\hline msh6 fun $30-K 603 A$ & $1.3 \times 10^{-7}\left(1.1 \times 10^{-7}-1.5 \times 10^{-7}\right)$ & 81 & $3.0 \times 10^{-5}\left(2.7 \times 10^{-5}-3.3 \times 10^{-5}\right)$ & 790 \\
\hline msh3s & $2.1 \times 10^{-8}\left(1.4 \times 10^{-8}-3.0 \times 10^{-8}\right)$ & 14 & $4.2 \times 10^{-7}\left(3.5 \times 10^{-7}-5.0 \times 10^{-7}\right)$ & 11 \\
\hline msh3s fun $30 \Delta$ & $3.6 \times 10^{-8}\left(2.6 \times 10^{-8}-4.8 \times 10^{-8}\right)$ & 23 & $8.9 \times 10^{-7}\left(7.7 \times 10^{-7}-1.0 \times 10^{-6}\right)$ & 24 \\
\hline$m s h 2 \Delta$ & $5.1 \times 10^{-6}\left(3.9 \times 10^{-6}-6.4 \times 10^{-6}\right)$ & 3300 & $3.8 \times 10^{-4}\left(3.1 \times 10^{-4}-4.5 \times 10^{-4}\right)$ & 10,000 \\
\hline msh2 $2 \Delta$ fun $30 \Delta$ & $6.2 \times 10^{-6}\left(4.9 \times 10^{-6}-7.7 \times 10^{-6}\right)$ & 3900 & $2.5 \times 10^{-4}\left(2.0 \times 10^{-4}-3.1 \times 10^{-4}\right)$ & 6800 \\
\hline exo1s & $6.8 \times 10^{-9}\left(4.8 \times 10^{-9}-9.1 \times 10^{-9}\right)$ & 4.3 & $1.8 \times 10^{-6}\left(1.4 \times 10^{-6}-2.2 \times 10^{-6}\right)$ & 47 \\
\hline exo1s fun30s & $7.3 \times 10^{-9}\left(5.2 \times 10^{-9}-9.6 \times 10^{-9}\right)$ & 4.6 & $1.1 \times 10^{-6}\left(8.3 \times 10^{-7}-1.4 \times 10^{-6}\right)$ & 30 \\
\hline exo1s msh6 & $3.3 \times 10^{-8}\left(2.5 \times 10^{-8}-4.1 \times 10^{-8}\right)$ & 21 & $1.8 \times 10^{-5}\left(1.5 \times 10^{-5}-2.0 \times 10^{-5}\right)$ & 470 \\
\hline exo1s msh6s fun30 & $2.3 \times 10^{-7}\left(2.0 \times 10^{-7}-2.6 \times 10^{-7}\right)$ & 150 & $4.6 \times 10^{-5}\left(4.1 \times 10^{-5}-5.0 \times 10^{-5}\right)$ & 1200 \\
\hline
\end{tabular}

The hom3-10 $\left(\mathrm{Thr}^{+}\right)$and lys2:insE-A14 $\left(\mathrm{Lys}^{+}\right)$reversion rates are presented, with $95 \%$ confidence intervals in parentheses. The fold increase of reversion rates over that of wild-type cells is shown.

simultaneously with $\mathrm{MSH6}$ or $\mathrm{MSH3}$, the reversion rates increased synergistically rather than additively. For instance, fun $30 \Delta$ increased the reversion rate by $\sim 12$-fold in the msh $6 \Delta$ background at hom 3 and approximately sixfold at lys2. fun $30 \Delta$ increased the reversion rates by approximately twofold in msh $3 \Delta$ cells at both loci, and this increase was much higher than the sum of the rates from every single mutant. Importantly, fun $30 \Delta$ did not increase the reversion rates in msh2 $\Delta$ cells. This is consistent with the idea that FUN30 contributes to a mutation avoidance pathway epistatic to $M S H 2$. The phenotype of the Walker A mutant of Fun30 (fun30-K603A) closely resembled that of fun $30 \Delta$, suggesting that an ATP-dependent function is required for Fun30 to suppress mutations. To test whether the genetic interaction is specific to Msh2-containing complexes, we combined fun $30 \Delta$ with exo1 14 , which also partially impairs MMR (Tishkoff et al. 1997; Amin et al. 2001). Critically, fun30 4 did not show a synergistic increase in the reversion rates with exo1 $\triangle$, and the genetic interaction between FUN3O and MSH6 was kept in the

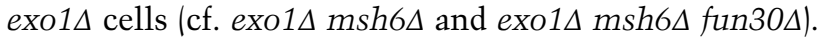
These factor-specific genetic interactions suggest that the function of Fun30 in MMR is related to MutSa and MutS $\beta$. As MutSa suppresses recombination between divergent sequences and since Fun30 is involved in the repair of DSBs (Chen et al. 2012; Costelloe et al. 2012; Eapen et al. 2012; Densham et al. 2016), we tested whether the observed genetic interactions are dependent on recombination (Supplemental Table S2). However, the synergistic effects were still observed in the homologous recombination-deficient rad52 $\Delta$ background (cf. rad52 2 msh $6 \Delta$ and

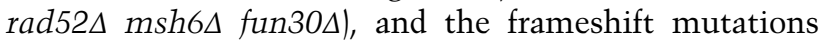
seen in fun $30 \Delta$ cells were more concentrated in the homopolymer "hot spot" runs, as seen in MMR mutants, than in rad52 $\Delta$ cells (Supplemental Fig. S7A,B). These data collectively suggest that an ATP-dependent function of Fun30 cooperates with the Msh2-containing complexes (especially with MutS $\beta$, at least for these genetic systems) to facilitate replication error correction. A temperature-sensitive mutant of FACT, spt16-d922 (Evans et al. 1998), did not show a significant mutator phenotype even when combined with msh64 (Supplemental Table S3). spt16d922 did not elevate the reversion rates also when combined with fun $30 \Delta$. As FACT is essential, we were not able to test the effect of fact $\Delta$, leaving open the question of whether FACT facilitates MMR in yeast. Given the functional overlap between the two factors in Xenopus, it is still possible that yeast FACT has a role redundant with Fun30 in MMR.

\section{Fun30 counteracts CAF-1 to assist Msh2-dependent MMR}

If Fun30 cooperates with the Msh2-containing complexes to counteract chromatin assembly, impairment of chromatin assembly should mitigate the mutator phenotype of fun $30 \Delta$. To test this possibility, we deleted $C A C 1$, the largest subunit of CAF-1. cac1 $\triangle$ alone slightly decreased the mutation rate at hom 3 and slightly increased the rate at lys2 (Table 2). The difference may be related to specific sequences or chromatin structure at two loci. Interestingly, however, in msh $6 \Delta$ fun $30 \Delta$ cells, cac1 $\Delta$ decreased the reversion rates by more than fivefold at hom3 and threefold at lys2. The reduction of reversion rates suggests that the majority of mutations seen in the msh64 fun304 double mutant is caused through CAF-1 function. cac1 $\Delta$ also reduced mutation rates in not only msh3 4 fun $30 \Delta$ cells but also $m s h 6 \Delta$ or $m s h 3 \Delta$ cells, suggesting that CAF-1 is inhibitory for both MutSa- and MutS $\beta$-dependent MMR. Importantly, cac1 $\Delta$ did not significantly change the reversion rates in msh2 $\Delta$ cells, implying that the effect of $\operatorname{cac} 1 \Delta$ is epistatic to $m s h 2 \Delta$. From these results, we infer that CAF-1 impedes Msh2dependent MMR, and Fun30, MutSa, and MutS $\beta$ counteract the function of CAF-1 to facilitate replication error correction. 
Table 2. The effect of cac1 deletion on reversion rates

\begin{tabular}{|c|c|c|c|c|}
\hline \multirow[b]{2}{*}{ Genotype } & \multicolumn{2}{|l|}{ hom3-10 } & \multicolumn{2}{|l|}{ lys2-14A } \\
\hline & Reversion rate & Fold increase & Reversion rate & Fold increase \\
\hline Wild type & $1.6 \times 10^{-9}\left(8.8 \times 10^{-10}-2.4 \times 10^{-9}\right)$ & 1.0 & $3.7 \times 10^{-8}\left(3.0 \times 10^{-8}-4.6 \times 10^{-8}\right)$ & 1.0 \\
\hline $\operatorname{cac} 1 \Delta^{\prime}$ & $1.3 \times 10^{-9}\left(6.7 \times 10^{-10}-2.0 \times 10^{-9}\right)$ & 0.8 & $5.8 \times 10^{-8}\left(4.5 \times 10^{-8}-7.2 \times 10^{-8}\right)$ & 1.6 \\
\hline fun30s & $3.3 \times 10^{-9}\left(2.2 \times 10^{-9}-4.7 \times 10^{-9}\right)^{\prime}$ & 2.1 & $7.0 \times 10^{-8}\left(5.8 \times 10^{-8}-8.2 \times 10^{-8}\right)$ & 1.9 \\
\hline fun $30 \Delta \operatorname{cac} 1 \Delta$ & $2.3 \times 10^{-9}\left(1.5 \times 10^{-9}-3.2 \times 10^{-9}\right)$ & 1.4 & $1.2 \times 10^{-7}\left(9.8 \times 10^{-8}-1.5 \times 10^{-7}\right)$ & 3.3 \\
\hline msh64 & $1.0 \times 10^{-8}\left(7.0 \times 10^{-9}-1.4 \times 10^{-8}\right)$ & 6.5 & $6.9 \times 10^{-6}\left(5.6 \times 10^{-6}-8.4 \times 10^{-6}\right)$ & 190 \\
\hline msh $6 \Delta \operatorname{cac} 1 \Delta$ & $6.3 \times 10^{-9}\left(3.9 \times 10^{-9}-9.2 \times 10^{-9}\right)$ & 4.0 & $5.6 \times 10^{-6}\left(4.3 \times 10^{-6}-7.0 \times 10^{-6}\right)$ & 150 \\
\hline msh6 fun $30 \Delta$ & $1.2 \times 10^{-7}\left(1.0 \times 10^{-7}-1.5 \times 10^{-7}\right)$ & 79 & $4.0 \times 10^{-5}\left(3.5 \times 10^{-5}-4.5 \times 10^{-5}\right)$ & 1100 \\
\hline msh6 6 fun $30 \Delta$ cac1s & $2.3 \times 10^{-8}\left(1.7 \times 10^{-8}-2.9 \times 10^{-8}\right)$ & 15 & $1.3 \times 10^{-5}\left(1.1 \times 10^{-5}-1.5 \times 10^{-5}\right)$ & 340 \\
\hline msh3s & $2.1 \times 10^{-8}\left(1.4 \times 10^{-8}-3.0 \times 10^{-8}\right)$ & 14 & $4.2 \times 10^{-7}\left(3.5 \times 10^{-7}-5.0 \times 10^{-7}\right)$ & 11 \\
\hline$m s h 3 \Delta \operatorname{cac} 1 \Delta$ & $1.1 \times 10^{-8}\left(6.8 \times 10^{-9}-1.6 \times 10^{-8}\right)$ & 7.0 & $3.2 \times 10^{-7}\left(2.7 \times 10^{-7}-3.8 \times 10^{-7}\right)$ & 8.6 \\
\hline$m s h 3 \Delta$ fun $30 \Delta$ & $3.6 \times 10^{-8}\left(2.6 \times 10^{-8}-4.8 \times 10^{-8}\right)$ & 23 & $8.9 \times 10^{-7}\left(7.7 \times 10^{-7}-1.0 \times 10^{-6}\right)$ & 24 \\
\hline msh3s fun $30 \Delta$ cac1s & $1.8 \times 10^{-8}\left(1.2 \times 10^{-8}-2.4 \times 10^{-8}\right)$ & 11 & $5.6 \times 10^{-7}\left(4.9 \times 10^{-7}-6.4 \times 10^{-7}\right)$ & 15 \\
\hline msh2s & $5.1 \times 10^{-6}\left(3.9 \times 10^{-6}-6.4 \times 10^{-6}\right)$ & 3300 & $3.8 \times 10^{-4}\left(3.1 \times 10^{-4}-4.5 \times 10^{-4}\right)$ & 10,000 \\
\hline $\operatorname{msh} 2 \Delta \operatorname{cac} 1 \Delta$ & $5.6 \times 10^{-6}\left(4.7 \times 10^{-6}-6.6 \times 10^{-6}\right)$ & 3500 & $3.1 \times 10^{-4}\left(2.7 \times 10^{-4}-3.6 \times 10^{-4}\right)$ & 8300 \\
\hline
\end{tabular}

The hom3-10 $\left(\mathrm{Thr}^{+}\right)$and lys2:insE-A14 $\left(\mathrm{Lys}^{+}\right)$reversion rates are presented, with $95 \%$ confidence intervals in parentheses. The fold increase of reversion rates over that of wild-type cells is shown. The rates of CAC1 cells were duplicated from Table 1.

\section{Discussion}

How the MMR machinery handles nucleosomes around mismatched bases is a key question for understanding the MMR reaction in the context of chromatin. Previous studies have shown that eukaryotic cells have multiple mechanisms to function on chromatinized DNA, which is shown to be inhibitory for MMR in vitro ( $\mathrm{Li}$ et al. 2009; Gorman et al. 2010; Schopf et al. 2012; Brown et al. 2016) but may not be so in vivo (Lujan et al. 2014). MutSa has a chromatin remodeling activity and interferes with CAF-1 function, likely to promote MMR on chromatin (Javaid et al. 2009; Kadyrova et al. 2011; Schopf et al. 2012; Rodriges Blanko et al. 2016). It is also possible that nucleosomes and other obstructions on DNA are not inhibitory for the MutLa step in MMR, as eukaryotic MutL $\alpha$ and bacterial MutL form a large proteinaceous ring that can bypass nucleosomes or MutS while traveling along DNA (Gorman et al. 2010; Liu et al. 2016). In this study, using Xenopus egg extracts that recapitulate chromatin assembly and MMR in vitro, we discovered that the eukaryotic MMR system has a remarkable ability to exclude nucleosomes around a mismatched base. We further showed that the SNF2 family chromatin remodeler Smarcadl assists both nucleosome exclusion and the repair of mismatches on chromatinized DNA. Genetic experiments in yeast provided evidence that the yeast homolog of Smarcad1 contributes to the MutSa- and MutS $\beta$-dependent MMR by counteracting the function of CAF-1. Both the biochemical and genetic data indicate that Smarcad1/Fun30 is an MMR accessory factor that assists the function of Msh2-containing mismatch sensor complexes.

Our data showed that nucleosome exclusion occurs at the step of Msh2-dependent mismatch recognition. Thus, Msh2, but not Mlh1, is required for exclusion of nucleosomes, and nucleosome exclusion occurs in the absence of strand discrimination signals. Consistent with this, chromatin loading of Smarcadl was dependent on Msh2 but not on Mlh1. It is noteworthy that we observed no detectable loading of Smarcad1 in our plasmid pulldown assay in the absence of a mismatch. This finding suggests that Smarcad1 is recruited to chromatin through the interaction with MMR proteins rather than with nucleosomes. Previous reports have also shown that the Smarcad1 homologs function at rather specific chromosomal loci such as the site of DSBs, heterochromatin, and centromeres (Neves-Costa et al. 2009; Stralfors et al. 2011; Chen et al. 2012; Costelloe et al. 2012; Eapen et al. 2012; Densham et al. 2016; Taneja et al. 2017). Although only a small portion of Smarcad1 was pulled down with Msh2 and Msh6 by immunoprecipitation (Fig. 4D), the amount of Smarcad1 on a mismatch-carrying DNA was comparable with that of Msh2 (Fig. 4C). As the MutS $\alpha$ and MutS $\beta$ complexes change their conformation upon the recognition of a mismatch, it would be possible that the ATP-bound form of MutS $\alpha$ - and also possibly MutS $\beta$ - preferentially interacts with Smarcad1 to load it onto DNA. We also showed that simultaneous depletion of Smarcadl and FACT further weakens nucleosome exclusion. Interestingly, FACT is a histone chaperone that promotes histone exchange (Formosa 2012), and yeast Fun30 also shows a histone exchange activity in vitro (Awad et al. 2010). Smarcadl and FACT might assist nucleosome exclusion by accelerating the turnover rate of histones. More mechanistic analyses are needed to clarify how these factors contribute to the exclusion of nucleosomes around a mismatch.

An important question is how nucleosome exclusion and Smarcadl contribute to post-replicative MMR. Our stepwise incubation experiments showed that, at least in a situation where nucleosomes are assembled around a mismatch before the initiation of MMR, Smarcad1 contributes to the repair of the mismatch (Fig. 7). This finding supports a hypothesis that Smarcadl collaborates with Msh2-containing complexes to promote the repair of mismatches by displacing local nucleosomes. However, at 
this point, it is difficult to estimate the contribution of nucleosome exclusion to in vivo MMR. Our biochemical data indicate that Smarcad1's contribution to nucleosome exclusion is partial, and, if this is also true in yeast, the mutator phenotype of fun30 4 may represent only a part of the contribution of nucleosome exclusion to MMR. Interestingly, in our experiments, recombinant MutSa restores gap-directed MMR (Kawasoe et al. 2016) but not nucleosome exclusion in Msh2-depleted NPE (Fig. 2B). This finding suggests that Msh2 depletion likely codepletes a factor that is critical for nucleosome exclusion. Since Smarcad1 is only partially required for nucleosome exclusion and since Smarcad1 is not quantitatively codepleted with Msh2, the factor that is codepleted with Msh2 would not be Smarcad1. Identification of such factors may clarify the relative contribution of nucleosome exclusion to MMR. Currently, however, it is still possible that our recombinant MutSa lacks some specific activity that is critical for nucleosome exclusion.

The genetic data in yeast were consistent with the biochemical data and at least partially compensated for limitations of the in vitro experiments. Thus, fun $30 \Delta \mathrm{ex}-$ acerbated the reversion rates in $m s h 6 \Delta$ and $m s h 3 \Delta$ cells but not in msh $2 \Delta$ cells, suggesting that Fun 30 cooperates with the Msh2-containing complexes to assist replication error correction. In addition, deletion of $C A C 1$ reduced the reversion rates in fun $30 \Delta$ msh6 cells, suggesting that Fun 30 has a role in counteracting CAF-1. This is in a good agreement with biochemical data in Xenopus; Smarcad1 facilitates mismatch correction on a chromatinized template (Fig. 7) and assists nucleosome exclusion by counteracting CAF-1-mediated chromatin assembly (Fig. 6). It should also be noted that our genetic experiments are in good agreement with the data showing that CAF-1 is inhibitory for MMR (Kadyrova et al. 2011; Schopf et al. 2012; Rodriges Blanko et al. 2016) and suppresses the cytotoxic activity of the MMR system upon treatment with a DNA-alkylating agent (Kadyrova et al. 2016). Curiously, the genetic data suggest that yeast Fun30 is more important for MutS $\beta$-dependent MMR than for MutSa-dependent MMR. Because MutSa has an ability to counteract CAF-1-mediated chromatin assembly (Javaid et al. 2009; Kadyrova et al. 2011; Schopf et al. 2012; Rodriges Blanko et al. 2016), it could be less dependent on Fun30 than MutS $\beta$, although it is not clear at this point whether MutS $\beta$ lacks such an ability. However, as we measured reversions at only two loci and detected exclusively -1 frameshifts, genome-wide evaluation of unbiased mutation rates and spectra in fun $30 \Delta$ cells would be essential for a comprehensive understanding of the Fun30 function in MMR. Likewise, although the genetic interaction between FUN30, MMR factors, and CAC1 is generally in good agreement with a scenario in which Fun30 counteracts CAF-1-mediated chromatin assembly to assist MMR, other possibilities are currently not excluded. Establishment of a biochemical assay with yeast proteins will be a key to connecting the biochemical data in Xenopus and the genetic data in yeast.

In conclusion, our study established that the Msh2-dependent MMR system has an ability to exclude nucleo- somes around mismatches and identified Smarcad1/ Fun30 as an accessory factor for nucleosome exclusion and Msh2-dependent mismatch correction. Although post-replicative MMR may occur mostly just behind the replication fork, it is possible that nucleosome exclusion becomes more important for MMR after the completion of chromatin assembly. In the leading strand, ribonucleotides embedded by polymerase $\varepsilon$ serve as strand discrimination signals after their conversion into singlestrand gaps by ribonucleotide excision repair (Ghodgaonkar et al. 2013; Lujan et al. 2013). Since ribonucleotide excision repair occurs after nascent DNA synthesis, ribonucleotide-induced MMR is more likely to conflict with nucleosomes and could be more dependent on nucleosome exclusion. Nucleosome exclusion may also be involved in other Msh2-related reactions such as heteroduplex rejection during recombination, apoptosis upon alkylating DNA damage, and somatic hypermutation in immune cells. Our study serves as a basis for future investigations into these interesting possibilities.

\section{Materials and methods}

Xenopus egg extract

The NPE of Xenopus eggs was prepared essentially as described previously (Lebofsky et al. 2009). See the Supplemental Material for detail. Xenopus laevis was purchased from Kato-S-kagaku and maintained and handled according to the animal care regulations at Osaka University and Kyushu University.

Yeast genetic analysis

All S. cerevisiae strains used in this study were derived from BY4741 and are listed in Supplemental Table S4. Mutation rates were estimated by fluctuation analysis using the Ma-Sandri-Sarkar (MSS) maximum likelihood method (Sarkar et al. 1992; Rosche and Foster 2000). Ninety-five percent confidence intervals were estimated based on the mutation rates obtained by the MSS method. For each replicate in the fluctuation analysis, a yeast culture was started from a single colony and grown to the stationary phase in $10 \mathrm{~mL}$ of yeast extract-peptone-dextrose medium plus adenine. Appropriate aliquots of cells were plated onto synthetic dextrose (SD) medium with amino acids lacking lysine or threonine to count $\mathrm{Lys}^{+}$or $\mathrm{Thr}^{+}$revertants and onto synthetic complete (SC) medium to count viable cells. For strains with very high mutation rates (strains carrying $m s h 2 \Delta, m s h 6 \Delta$, or exo14), a single colony was directly suspended in $1 \mathrm{~mL}$ of distilled water, and appropriate aliquots were plated on solid medium. See the Supplemental Material for detail of the statistical testing.

Supercoiling, gap-directed $M M R$, and primer extension assays

The supercoiling assay was carried out essentially as described previously (Kawasoe et al. 2016). NPE was supplemented with $2 \mathrm{mM}$ ATP, $20 \mathrm{mM}$ phosphocreatine (PC), and $5 \mu \mathrm{g} / \mathrm{mL}$ creatine phosphokinase $(\mathrm{CPK})$ and preincubated for $5 \mathrm{~min}$ at $22^{\circ} \mathrm{C}$. A typical reaction consisted of $17.4 \mu \mathrm{L}$ of NPE, $0.2 \mu \mathrm{L}$ of $200 \mathrm{mM}$ ATP, $0.4 \mu \mathrm{L}$ of $1 \mathrm{M} \mathrm{PC}, 0.02 \mu \mathrm{L}$ of $5 \mathrm{mg} / \mathrm{mL}$ CPK, and $2 \mu \mathrm{L}$ of substrate DNA $(200 \mathrm{ng} / \mu \mathrm{L}$ in TE: $10 \mathrm{mM}$ Tris- $\mathrm{HCl}, 1 \mathrm{mM}$ ethylenediaminetetraacetic acid [EDTA] at $\mathrm{pH}$ 7.4). Gap-carrying DNA was used at a final concentration of $20 \mathrm{ng} / \mu \mathrm{L}$ for the gap-directed MMR assay, and primed ssDNA was used at a final concentration 
of $10 \mathrm{ng} / \mu \mathrm{L}$ for the primer extension assay. After adding DNA, reaction mixtures were incubated at $22^{\circ} \mathrm{C}$, and aliquots $(1.5-3 \mu \mathrm{L}$ for most experiments) were stopped by addition of $100 \mu \mathrm{L}$ of $1 \%$ SDS in 20 mM EDTA. DNA was purified by proteinase $\mathrm{K}$ treatment, phenol/chloroform extraction, and ethanol precipitation. To analyze the MMR efficiency, 10 ng of DNA was digested with XmnI and BamHI-HF or XhoI (New England Biolabs) in a 10$\mu \mathrm{L}$ reaction. In the primer extension assay, 30 ng of DNA was digested with $3 \mathrm{U}$ of S1 nuclease (Takara) and $0.3 \mathrm{U}$ of ExoV (New England Biolabs) or with $3 \mathrm{U}$ of XhoI, $3 \mathrm{U}$ of S1 nuclease, and $0.3 \mathrm{U}$ of $\lambda$ exonuclease (New England Biolabs) in $1 \times$ CutSmart buffer (New England Biolabs) in an $8-\mu \mathrm{L}$ reaction. After agarose gel electrophoresis, DNA was stained with SYBR Gold nucleic acid stain (Life Technologies) and scanned with a Typhoon FLA9000 (GE Healthcare) or ChemiDoc Touch (Bio-Rad). Signal intensities were quantified using ImageJ (National Institutes of Health).

MNase digestion, Southern blotting, and $q P C R$

A $17-\mu \mathrm{L}$ supercoiling reaction was set up and incubated for $60 \mathrm{~min}$ at $22^{\circ} \mathrm{C}$. A $2-\mu \mathrm{L}$ aliquot was sampled for supercoiling, and another $15-\mu \mathrm{L}$ aliquot was quickly diluted with $1.5 \mathrm{~mL}$ of MNase buffer (10 mM Tris- $\mathrm{HCl}, 50 \mathrm{mM} \mathrm{NaCl}, 2.5 \mathrm{mM} \mathrm{CaCl}_{2}$ at $\mathrm{pH} 7.4$ ) containing $20 \mathrm{U} / \mathrm{mL}$ MNase (Worthington). The samples were incubated at $37^{\circ} \mathrm{C} ; 350 \mu \mathrm{L}$ each of aliquots was stopped by addition of $50 \mu \mathrm{L}$ of C-stop buffer (160 mM EDTA, 6.8\% SDS) at 15, 30, 60, and $120 \mathrm{sec}$; and DNA was purified. For Southern blotting, DNA was separated on $1.2 \%$ agarose gel in $0.5 \times$ TBE (Tris-borate-EDTA) buffer, stained with SYBR Gold, and scanned with a Typhoon FLA9000. DNA was then transferred onto Hybond $\mathrm{N}^{+}$nylon membrane (GE Healthcare) and hybridized with a ${ }^{32} \mathrm{P}$-labeled probe prepared from the PvuII-PvuII 473-base-pair (bp) fragment of pMM1 using the random primer DNA labeling kit (Takara). The probe was stripped off after detection of ${ }^{32} \mathrm{P}$, and the membrane was rehybridized with another probe prepared from the DraI-DraI 692-bp fragment. $\beta$ Rays from ${ }^{32} \mathrm{P}$ were detected by a Typhoon FLA9000 using a phosphorimaging plate. For qPCR, supercoiling reactions were set up in the presence of $5 \mathrm{ng} / \mathrm{\mu L}$ pControl/pCDFDuet-1 (Merck Millipore). DNA samples were diluted in $\mathrm{TE}$, and $10-\mu \mathrm{L}$ reactions (7 $\mu \mathrm{L}$ of qPCR master mix, $2 \mu \mathrm{L}$ of $1 \mu \mathrm{M}$ primer mix, and $1 \mu \mathrm{L}$ of diluted DNA) were run in a Mx3000P system (Stratagene) using KOD SYBR qPCR mix (Toyobo) and primers listed in Supplemental Table S5.

Plasmid pull-down and mass spectrometry identification of DNA-bound proteins

Singly biotinylated plasmid DNA was immobilized on streptavidin-coated biotin-Sepharose beads as described previously (Kawasoe et al. 2016). Immobilized DNA was incubated in NPE at $20 \mathrm{ng} / \mu \mathrm{L}$ (600 ng of DNA bound to $6 \mu \mathrm{L}$ of Sepharose in a $30-\mu \mathrm{L}$ reaction) for $30 \mathrm{~min}$ at $22^{\circ} \mathrm{C}$. The reaction mixture was diluted with $200 \mu \mathrm{L}$ of $1 \times$ egg lysis buffer (ELB; $10 \mathrm{mM}$ HEPES-KOH, $2.5 \mathrm{mM} \mathrm{MgCl}_{2}, 50 \mathrm{mM} \mathrm{KCl}$ at $\mathrm{pH}$ 7.7) containing $0.2 \%$ Triton $\mathrm{X}-100$, layered over $300 \mu \mathrm{L}$ of ELB containing $500 \mathrm{mM}$ sucrose, and centrifuged at $12,700 \mathrm{~g}$ for $2 \mathrm{~min}$ at $4^{\circ} \mathrm{C}$ in a horizontal centrifuge (Tomy Seiko). The beads were washed three times with ELB, and bound proteins were eluted with $12 \mu \mathrm{L}$ of Laemmli's SDS sample buffer $162.5 \mathrm{mM}$ Tris-HCl, 10\% glycerol, 3\% SDS, $0.005 \%$ bromophenol blue, 5\% 2-mercaptoethanol at $\mathrm{pH} 6.8$ ). To monitor DNA recovery, DNA was extracted with phenol/ chloroform, precipitated with ethanol, and dissolved in TE. The amount of DNA was determined by qPCR with primers 1842 and 1843. Mass spectrometry analysis was carried out as de- scribed previously with minor modifications (Nozawa et al. 2010). The LC-MS/MS data were searched against a $X$. laevis subset database created from RefSeq (release 82). Identified proteins were semiquantified by spectral counting (Liu et al. 2004) using Scaffold software version 4.8.3 (Proteome Software, Inc.).

Stepwise incubation assay

Immobilized DNA was incubated in NPE as described in the method for plasmid pull-down. After a 30-min incubation, the DNA was recovered by centrifugation in a benchtop centrifuge, washed three times with ELB, and incubated in the second NPE at $20 \mathrm{ng} / \mu \mathrm{L}$ concentration (100 ng of DNA bound to $1 \mu \mathrm{L}$ of Sepharose in a $5-\mu \mathrm{L}$ reaction) for $30 \mathrm{~min}$ at $22^{\circ} \mathrm{C}$ unless stated otherwise. For the experiment shown in Figure $2 \mathrm{G}$, biotin-free DNA was used as a substrate, and an equal volume of the second NPE was added directly to the reaction. The reaction was stopped by addition of $100 \mu \mathrm{L}$ of $1 \%$ SDS in 20 mM EDTA. DNA was purified by proteinase $\mathrm{K}$ treatment, phenol/chloroform extraction, and ethanol precipitation.

\section{Acknowledgments}

We thank Johannes Walter for antibodies and critical reading of the manuscript; Toshiki Tsurimoto and Eiji Ohashi for critical reading of the manuscript; Haruhiko Takisawa, Yumiko Kubota, Masato Kanemaki, Hiroshi Kimura, Ruibin Zhu, Mari Iwabuchi, and Keita Ohsumi for antibodies and cDNAs; Chikako Tokumura for maintenance of insect cells; and Naomi Takagi for mass spectrometry. This work was supported by grants from the Ministry of Education, Culture, Sports, and Technologies (MEXT) in Japan to T.S.T. (JP16K14671, JP25131712, JP25650011, JP25711022, JP23131507, JP23657114, JP17H01876, and JP18H04716), K.N. (JP15K06942, JP15H01462, and JP17H06426), Y.K. (JP17H069 35), and C.O. (JP16H04739 and JP25116004); the Naito Foundation, the Inamori Foundation, the Uehara Memorial Foundation, the Mochida Memorial Foundation for Medical and Pharmaceutical Research, and the Takeda Science Foundation to T.S.T.; and the Uehara Memorial Foundation and Institute for Fermentation, Osaka (IFO) to S.T. R.T. was a predoctoral research fellow of the Japan Society for the Promotion of Science.

Author contributions: T.L.H first discovered that a mismatchcarrying plasmid does not efficiently supercoil in NPE. K.T. prepared recombinant MutS $\alpha$ and found that nucleosome exclusion depends on Msh2. K.N. and C.O. performed the mass spectrometry analysis. S.T. provided yeast strains and helped with yeast experiments. H.M. and T.N. contributed to the design of experiments and data interpretation. R.T. performed most experiments. Y.K. performed the experiments in Figure 7. T.S.T. and R.T. designed the experiments, analyzed the data, and drafted the paper. All authors edited and approved the paper.

\section{References}

Amin NS, Nguyen MN, Oh S, Kolodner RD. 2001. exo1-dependent mutator mutations: model system for studying functional interactions in mismatch repair. Mol Cell Biol 21: 51425155.

Awad S, Ryan D, Prochasson P, Owen-Hughes T, Hassan AH. 2010. The Snf2 homolog Fun30 acts as a homodimeric ATPdependent chromatin-remodeling enzyme. J Biol Chem 285: 9477-9484.

Brown MW, Kim Y, Williams GM, Huck JD, Surtees JA, Finkelstein IJ. 2016. Dynamic DNA binding licenses a repair factor 
to bypass roadblocks in search of DNA lesions. Nat Commun 7: 10607.

Chen X, Cui D, Papusha A, Zhang X, Chu CD, Tang J, Chen K, Pan X, Ira G. 2012. The Fun30 nucleosome remodeller promotes resection of DNA double-strand break ends. Nature 489: 576-580.

Chen Z, Tran M, Tang M, Wang W, Gong Z, Chen J. 2016. Proteomic analysis reveals a novel mutator $S$ (MutS) partner involved in mismatch repair pathway. Mol Cell Proteomics 15: 1299-1308.

Costelloe T, Louge R, Tomimatsu N, Mukherjee B, Martini E, Khadaroo B, Dubois K, Wiegant WW, Thierry A, Burma S, et al. 2012. The yeast Fun30 and human SMARCAD1 chromatin remodellers promote DNA end resection. Nature 489: 581-584.

Densham RM, Garvin AJ, Stone HR, Strachan J, Baldock RA, Daza-Martin M, Fletcher A, Blair-Reid S, Beesley J, Johal B, et al. 2016. Human BRCA1-BARD1 ubiquitin ligase activity counteracts chromatin barriers to DNA resection. Nat Struct Mol Biol 23: 647-655.

Drummond JT, Genschel J, Wolf E, Modrich P. 1997. DHFR/ MSH3 amplification in methotrexate-resistant cells alters the hMutSa/hMutS $\beta$ ratio and reduces the efficiency of basebase mismatch repair. Proc Natl Acad Sci 94: 10144-10149.

Eapen VV, Sugawara N, Tsabar M, Wu WH, Haber JE. 2012. The Saccharomyces cerevisiae chromatin remodeler Fun30 regulates DNA end resection and checkpoint deactivation. Mol Cell Biol 32: 4727-4740.

Evans DR, Brewster NK, Xu Q, Rowley A, Altheim BA, Johnston GC, Singer RA. 1998. The yeast protein complex containing cdc68 and pob3 mediates core-promoter repression through the cdc68 N-terminal domain. Genetics 150: 1393-1405.

Formosa T. 2012. The role of FACT in making and breaking nucleosomes. Biochim Biophys Acta 1819: 247-255.

Gaillard PH, Martini EM, Kaufman PD, Stillman B, Moustacchi E, Almouzni G. 1996. Chromatin assembly coupled to DNA repair: a new role for chromatin assembly factor I. Cell 86: 887-896.

Genschel J, Littman SJ, Drummond JT, Modrich P. 1998. Isolation of MutS $\beta$ from human cells and comparison of the mismatch repair specificities of MutS $\beta$ and MutSa. I Biol Chem 273: 19895-19901.

Ghodgaonkar MM, Lazzaro F, Olivera-Pimentel M, Artola-Boran M, Cejka P, Reijns MA, Jackson AP, Plevani P, Muzi-Falconi M, Jiricny J. 2013. Ribonucleotides misincorporated into DNA act as strand-discrimination signals in eukaryotic mismatch repair. Mol Cell 50: 323-332.

Gorman J, Plys AJ, Visnapuu ML, Alani E, Greene EC. 2010. Visualizing one-dimensional diffusion of eukaryotic DNA repair factors along a chromatin lattice. Nat Struct Mol Biol 17: 932-938.

Haye JE, Gammie AE. 2015. The eukaryotic mismatch recognition complexes track with the replisome during DNA synthesis. PLoS Genet 11: e1005719.

Holmes J Jr, Clark S, Modrich P. 1990. Strand-specific mismatch correction in nuclear extracts of human and Drosophila melanogaster cell lines. Proc Natl Acad Sci 87: 5837-5841.

Hombauer H, Campbell CS, Smith CE, Desai A, Kolodner RD. 2011. Visualization of eukaryotic DNA mismatch repair reveals distinct recognition and repair intermediates. Cell 147: 1040-1053.

Iyer RR, Pluciennik A, Burdett V, Modrich PL. 2006. DNA mismatch repair: functions and mechanisms. Chem Rev 106: 302-323.
Javaid S, Manohar M, Punja N, Mooney A, Ottesen JJ, Poirier MG, Fishel R. 2009. Nucleosome remodeling by hMSH2-hMSH6. Mol Cell 36: 1086-1094.

Jiricny J. 2013. Postreplicative mismatch repair. Cold Spring Harb Perspect Biol 5: a012633.

Kadyrov FA, Dzantiev L, Constantin N, Modrich P. 2006. Endonucleolytic function of MutLa in human mismatch repair. Cell 126: 297-308.

Kadyrova LY, Blanko ER, Kadyrov FA. 2011. CAF-I-dependent control of degradation of the discontinuous strands during mismatch repair. Proc Natl Acad Sci 108: 2753-2758.

Kadyrova LY, Dahal BK, Kadyrov FA. 2016. The major replicative histone chaperone CAF-1 suppresses the activity of the DNA mismatch repair system in the cytotoxic response to a DNAmethylating agent. J Biol Chem 291: 27298-27312.

Kawasoe Y, Tsurimoto T, Nakagawa T, Masukata H, Takahashi TS. 2016. MutSa maintains the mismatch repair capability by inhibiting PCNA unloading. Elife 5: e15155.

Kleczkowska HE, Marra G, Lettieri T, Jiricny J. 2001. hMSH3 and hMSH6 interact with PCNA and colocalize with it to replication foci. Genes Dev 15: 724-736.

Kunkel TA, Erie DA. 2015. Eukaryotic mismatch repair in relation to DNA replication. Annu Rev Genet 49: 291-313.

Lebofsky R, Takahashi T, Walter JC. 2009. DNA replication in nucleus-free Xenopus egg extracts. Methods Mol Biol 521: 229-252.

Lee JB, Cho WK, Park J, Jeon Y, Kim D, Lee SH, Fishel R. 2014. Single-molecule views of MutS on mismatched DNA. DNA Repair (Amst) 20: 82-93.

Li F, Tian L, Gu L, Li GM. 2009. Evidence that nucleosomes inhibit mismatch repair in eukaryotic cells. J Biol Chem 284: 33056-33061.

Li F, Mao G, Tong D, Huang J, Gu L, Yang W, Li GM. 2013. The histone mark $\mathrm{H} 3 \mathrm{~K} 36 \mathrm{me} 3$ regulates human DNA mismatch repair through its interaction with MutSa. Cell 153: 590-600.

Liu H, Sadygov RG, Yates JR III. 2004. A model for random sampling and estimation of relative protein abundance in shotgun proteomics. Anal Chem 76: 4193-4201.

Liu J, Hanne J, Britton BM, Bennett J, Kim D, Lee JB, Fishel R. 2016. Cascading MutS and MutL sliding clamps control DNA diffusion to activate mismatch repair. Nature 539: 583-587.

Lucchini R, Sogo JM. 1995. Replication of transcriptionally active chromatin. Nature 374: 276-280.

Lujan SA, Williams JS, Clausen AR, Clark AB, Kunkel TA. 2013. Ribonucleotides are signals for mismatch repair of leadingstrand replication errors. Mol Cell 50: 437-443.

Lujan SA, Clausen AR, Clark AB, MacAlpine HK, MacAlpine DM, Malc EP, Mieczkowski PA, Burkholder AB, Fargo DC, Gordenin DA, et al. 2014. Heterogeneous polymerase fidelity and mismatch repair bias genome variation and composition. Genome Res 24: 1751-1764.

Lynch HT, Snyder CL, Shaw TG, Heinen CD, Hitchins MP. 2015. Milestones of Lynch syndrome: 1895-2015. Nat Rev Cancer 15: $181-194$.

Marra G, Iaccarino I, Lettieri T, Roscilli G, Delmastro P, Jiricny J. 1998. Mismatch repair deficiency associated with overexpression of the MSH3 gene. Proc Natl Acad Sci 95: 8568-8573.

Marsischky GT, Filosi N, Kane MF, Kolodner R. 1996. Redundancy of Saccharomyces cerevisiae MSH3 and MSH6 in MSH2dependent mismatch repair. Genes Dev 10: 407-420.

McKnight SL, Miller OL Jr. 1977. Electron microscopic analysis of chromatin replication in the cellular blastoderm Drosophila melanogaster embryo. Cell 12: 795-804. 
Narlikar GJ, Sundaramoorthy R, Owen-Hughes T. 2013. Mechanisms and functions of ATP-dependent chromatin-remodeling enzymes. Cell 154: 490-503.

Neves-Costa A, Will WR, Vetter AT, Miller JR, Varga-Weisz P. 2009. The SNF2-family member Fun 30 promotes gene silencing in heterochromatic loci. PLoS One 4: e8111.

Nozawa RS, Nagao K, Masuda HT, Iwasaki O, Hirota T, Nozaki N, Kimura H, Obuse C. 2010. Human POGZ modulates dissociation of HPla from mitotic chromosome arms through Aurora B activation. Nat Cell Biol 12: 719-727.

Okazaki N, Ikeda S, Ohara R, Shimada K, Yanagawa T, Nagase T, Ohara O, Koga H. 2008. The novel protein complex with SMARCAD1/KIAA1122 binds to the vicinity of TSS. I Mol Biol 382: 257-265.

Olivera Harris M, Kallenberger L, Artola Boran M, Enoiu M, Costanzo V, Jiricny J. 2015. Mismatch repair-dependent metabolism of $\mathrm{O}^{6}$-methylguanine-containing DNA in Xenopus laevis egg extracts. DNA Repair (Amst) 28: 1-7.

Pluciennik A, Dzantiev L, Iyer RR, Constantin N, Kadyrov FA, Modrich P. 2010. PCNA function in the activation and strand direction of MutLa endonuclease in mismatch repair. Proc Natl Acad Sci 107: 16066-16071.

Pluciennik A, Burdett V, Baitinger C, Iyer RR, Shi K, Modrich P. 2013. Extrahelical (CAG)/(CTG) triplet repeat elements support proliferating cell nuclear antigen loading and MutLa endonuclease activation. Proc Nat1 Acad Sci 110: 12277-12282.

Polo SE, Almouzni G. 2015. Chromatin dynamics after DNA damage: the legacy of the access-repair-restore model. DNA Repair (Amst) 36: 114-121.

Ransom M, Dennehey BK, Tyler JK. 2010. Chaperoning histones during DNA replication and repair. Cell 140: 183-195.

Ray-Gallet D, Quivy JP, Scamps C, Martini EM, Lipinski M, Almouzni G. 2002. HIRA is critical for a nucleosome assembly pathway independent of DNA synthesis. Mol Cell 9: 1091-1100.

Rodriges Blanko E, Kadyrova LY, Kadyrov FA. 2016. DNA mismatch repair interacts with CAF-1- and ASF1A-H3-H4-dependent histone $(\mathrm{H} 3-\mathrm{H} 4)^{2}$ tetramer deposition. J Biol Chem 291: 9203-9217.

Rosche WA, Foster PL. 2000. Determining mutation rates in bacterial populations. Methods 20: 4-17.

Rowbotham SP, Barki L, Neves-Costa A, Santos F, Dean W, Hawkes N, Choudhary P, Will WR, Webster J, Oxley D, et al. 2011. Maintenance of silent chromatin through replication requires SWI/SNF-like chromatin remodeler SMARCAD1. Mol Cell 42: 285-296.

Sarkar S, Ma WT, Sandri GH. 1992. On fluctuation analysis: a new, simple and efficient method for computing the expected number of mutants. Genetica 85: 173-179.

Schopf B, Bregenhorn S, Quivy JP, Kadyrov FA, Almouzni G, Jiricny J. 2012. Interplay between mismatch repair and chromatin assembly. Proc Natl Acad Sci 109: 1895-1900.
Shibahara K, Stillman B. 1999. Replication-dependent marking of DNA by PCNA facilitates CAF-1-coupled inheritance of chromatin. Cell 96: 575-585.

Sirbu BM, McDonald WH, Dungrawala H, Badu-Nkansah A, Kavanaugh GM, Chen Y, Tabb DL, Cortez D. 2013. Identification of proteins at active, stalled, and collapsed replication forks using isolation of proteins on nascent DNA (iPOND) coupled with mass spectrometry. I Biol Chem 288: 31458-31467.

Smith S, Stillman B. 1989. Purification and characterization of CAF-I, a human cell factor required for chromatin assembly during DNA replication in vitro. Cell 58: 15-25.

Smith DJ, Whitehouse I. 2012. Intrinsic coupling of laggingstrand synthesis to chromatin assembly. Nature 483: 434-438.

Sogo JM, Stahl H, Koller T, Knippers R. 1986. Structure of replicating simian virus 40 minichromosomes. The replication fork, core histone segregation and terminal structures. J Mol Biol 189: 189-204.

Stralfors A, Walfridsson J, Bhuiyan H, Ekwall K. 2011. The FUN30 chromatin remodeler, Fft3, protects centromeric and subtelomeric domains from euchromatin formation. PLoS Genet 7: e1001334.

Taneja N, Zofall M, Balachandran V, Thillainadesan G, Sugiyama T, Wheeler D, Zhou M, Grewal SI. 2017. SNF2 family protein $\mathrm{Fft} 3$ suppresses nucleosome turnover to promote epigenetic inheritance and proper replication. Mol Cell 66: 50-62.e6.

Thomas DC, Roberts JD, Kunkel TA. 1991. Heteroduplex repair in extracts of human HeLa cells. I Biol Chem 266: 3744-3751.

Tishkoff DX, Boerger AL, Bertrand P, Filosi N, Gaida GM, Kane MF, Kolodner RD. 1997. Identification and characterization of Saccharomyces cerevisiae EXO1, a gene encoding an exonuclease that interacts with MSH2. Proc Natl Acad Sci 94: 7487-7492.

Tran HT, Keen JD, Kricker M, Resnick MA, Gordenin DA. 1997. Hypermutability of homonucleotide runs in mismatch repair and DNA polymerase proofreading yeast mutants. Mol Cell Biol 17: 2859-2865.

Walter J, Newport J. 2000. Initiation of eukaryotic DNA replication: origin unwinding and sequential chromatin association of Cdc45, RPA, and DNA polymerase a. Mol Cell 5: 617-627.

Walter J, Sun L, Newport J. 1998. Regulated chromosomal DNA replication in the absence of a nucleus. Mol Cell 1: 519-529.

Yuan F, Gu L, Guo S, Wang C, Li GM. 2004. Evidence for involvement of HMGB1 protein in human DNA mismatch repair. I Biol Chem 279: 20935-20940.

Zhang Y, Yuan F, Wang D, Gu L, Li GM. 2008. Identification of regulatory factor $\mathrm{X}$ as a novel mismatch repair stimulatory factor. J Biol Chem 283: 12730-12735. 


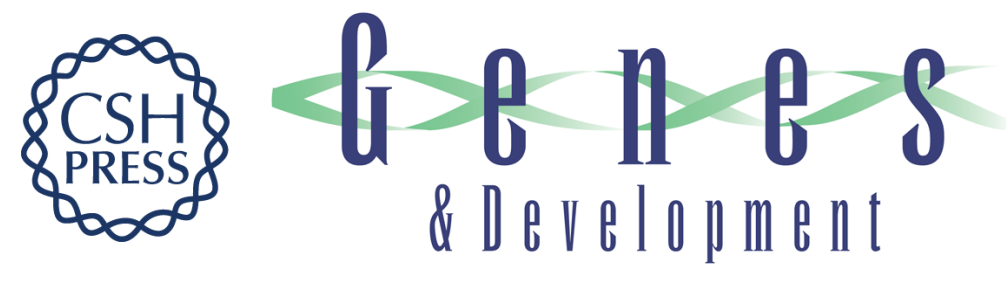

\section{Nucleosomes around a mismatched base pair are excluded via an Msh2-dependent reaction with the aid of SNF2 family ATPase Smarcad1}

Riki Terui, Koji Nagao, Yoshitaka Kawasoe, et al.

Genes Dev. 2018, 32: originally published online June 13, 2018

Access the most recent version at doi:10.1101/gad.310995.117

Supplemental Material

References

Creative

Commons

License

Email Alerting

Service
http://genesdev.cshlp.org/content/suppl/2018/06/13/gad.310995.117.DC1

This article cites 69 articles, 26 of which can be accessed free at: http://genesdev.cshlp.org/content/32/11-12/806.full.html\#ref-list-1

This article, published in Genes \& Development, is available under a Creative Commons License (Attribution 4.0 International), as described at http://creativecommons.org/licenses/by/4.0/.

Receive free email alerts when new articles cite this article - sign up in the box at the top right corner of the article or click here.

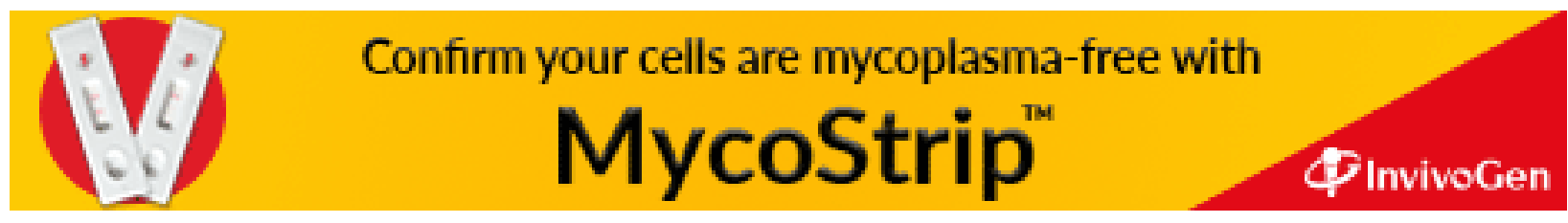

